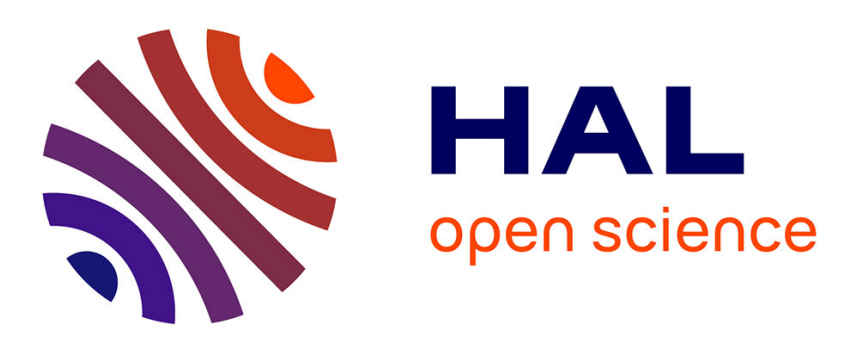

\title{
A Hybrid High-Order discretization combined with Nitsche's method for contact and Tresca friction in small strain elasticity
}

Franz Chouly, Alexandre Ern, Nicolas Pignet

\section{- To cite this version:}

Franz Chouly, Alexandre Ern, Nicolas Pignet. A Hybrid High-Order discretization combined with Nitsche's method for contact and Tresca friction in small strain elasticity. SIAM Journal on Scientific Computing, 2020, 42, pp.2300-2324. 10.1137/19M1286499 . hal-02283418v2

HAL Id: hal-02283418

https://hal.science/hal-02283418v2

Submitted on 26 Feb 2020

HAL is a multi-disciplinary open access archive for the deposit and dissemination of scientific research documents, whether they are published or not. The documents may come from teaching and research institutions in France or abroad, or from public or private research centers.
L'archive ouverte pluridisciplinaire HAL, est destinée au dépôt et à la diffusion de documents scientifiques de niveau recherche, publiés ou non, émanant des établissements d'enseignement et de recherche français ou étrangers, des laboratoires publics ou privés. 


\title{
A Hybrid High-Order discretization combined with Nitsche's method for contact and Tresca friction in small strain elasticity
}

\author{
Franz Chouly*,2, Alexandre Ern ${ }^{3}$, and Nicolas Pignet ${ }^{1,3}$ \\ ${ }^{1}$ EDF R\&D / ERMES, Palaiseau, France and IMSIA - UMR EDF/CNRS/CEA/ENSTA \\ 9219, Palaiseau, France \\ ${ }^{2}$ Université Bourgogne Franche-Comté, Institut de Mathématiques de Bourgogne, 21078 \\ Dijon, France \\ ${ }^{3}$ Université Paris-Est, CERMICS (ENPC), 77455 Marne-la-Vallée Cedex 2, France and \\ INRIA Paris, 75589 Paris, France
}

February 26, 2020

\begin{abstract}
We devise and analyze a Hybrid high-order (HHO) method to discretize unilateral and bilateral contact problems with Tresca friction in small strain elasticity. The nonlinear frictional contact conditions are enforced weakly by means of a consistent Nitsche's technique with symmetric, incomplete, and skewsymmetric variants. The present HHO-Nitsche method supports polyhedral meshes and delivers optimal energy-error estimates for smooth solutions under some minimal thresholds on the penalty parameters for all the symmetry variants. An explicit tracking of the dependency of the penalty parameters on the material coefficients is carried out to identify the robustness of the method in the incompressible limit, showing the more advantageous properties of the skew-symmetric variant. 2D and $3 \mathrm{D}$ numerical results including comparisons to benchmarks from the literature and to solutions obtained with an industrial software, as well as a prototype for an industrial application, illustrate the theoretical results and reveal that in practice the method behaves in a robust manner for all the symmetry variants in Nitsche's formulation.
\end{abstract}

Keywords. General meshes; Arbitrary order; Hybrid discretization; Nitsche's method; Unilateral contact; Tresca friction; Elasticity; Locking-free methods.

Mathematics Subject Classification. 65N12, 65N30, 74M15.

\section{Introduction}

Hybrid high-order (HHO) methods have been introduced for linear elasticity in [22] and for linear diffusion problems in [23]. HHO methods are formulated in terms of face unknowns that are polynomials of arbitrary order $k \geq 0$ on each mesh face and in terms of cell unknowns which are polynomials of order $l \in\{k, k \pm 1\}$, with $l \geq 0$, in each mesh cell. The devising of HHO methods hinges on two operators, both defined locally in each mesh cell: a reconstruction operator and a stabilization operator. The cell unknowns can be eliminated locally by static condensation leading to a global transmission problem posed solely in terms of the face unknowns. HHO methods offer various assets: they support polyhedral meshes, lead to local conservation principles, and optimal convergence rates. HHO methods have been bridged in [18] to Hybridizable Discontinuous Galerkin methods [19] and to nonconforming Virtual Element Methods [5]. HHO methods have been extended to many other PDEs. Examples in computational mechanics include nonlinear elasticity [7], hyperelasticity with finite deformations [2], and elastoplasticity with small [4] and finite [3] deformations.

\footnotetext{
*franz.chouly@u-bourgogne.fr
} 
The goal of the present work is to devise, analyze and evaluate numerically a HHO method to approximate contact problems with Tresca friction in small strain elasticity. Either unilateral or bilateral contact can be considered. The main ingredient is to employ a Nitsche-type formulation to enforce the nonlinear frictional contact conditions. The present HHO-Nitsche method can be deployed on polyhedral meshes. As is classical with Nitsche's technique, we can consider symmetric, incomplete and skew-symmetric variants. Our main results, Theorem 4.6 and Corollary 4.8, provide for all symmetry variants quasi-optimal energy error estimates with convergence rates of order $\mathcal{O}\left(h^{r}\right)$ for solutions with regularity $H^{1+r}, r \in\left(\frac{1}{2}, k+1\right]$, where $h$ is the mesh size and $k \geq 1$ is the order of the polynomials for the cell and the face unknowns, except for the face unknowns located on the frictional contact boundary where polynomials of order $(k+1)$ are employed. Note that the optimal order of convergence is $\mathcal{O}\left(h^{k+1}\right)$ obtained with $r=k+1$. These results are established under minimal thresholds for the penalty parameters weakly enforcing the contact and friction conditions, and do not require any assumption on the (a priori unknown) friction/contact set. Particular attention in the analysis is paid to the dependency of these parameters on the Lamé parameters, showing that the skewsymmetric variant enjoys more favorable properties regarding robustness in the incompressible limit, at least from a theoretical viewpoint. Our $2 \mathrm{D}$ and $3 \mathrm{D}$ numerical tests include comparisons with benchmarks from the literature and with solutions obtained with the industrial software code_aster [26]. We also consider a prototype of an industrial application featuring a notched plug in a rigid pipe. Our numerical tests indicate a more favorable dependency of the penalty parameters on the material parameters since robustness in the quasi-incompressible regime is observed in all considered situations.

Let us put our work in perspective with the literature. For most discretizations, Tresca friction creates additional difficulties in order to establish optimal convergence in comparison to the frictionless case (see, e.g., $[34,29,25]$ and the references therein for frictionless contact). As a consequence convergence results addressing Tresca friction are quite rare. The rate $\mathcal{O}\left(h^{r}\right)$ for the energy error with a regularity $H^{1+r}(\Omega)$, $r \in(0,1]$, has been obtained in the 2D case for a mixed low-order finite element method (FEM) under some technical assumptions on the contact/friction set [34, Theorem 4.9] (this is the first optimal bound to the best of our knowledge). In the $3 \mathrm{D}$ case the rate $O\left(h^{\min \left(\frac{1}{2}, r\right)}\right)$ has also been reached without additional assumption [34, Theorem 4.10]. For the penalty method, the rate of $\mathcal{O}\left(h^{\frac{1}{2}+\frac{r}{2}+r^{2}}\right)$ with a regularity $H^{\frac{3}{2}+r}(\Omega)$, $r \in\left(0, \frac{1}{2}\right)$, and the quasi-optimal rate of $\mathcal{O}\left(h|\log h|^{\frac{1}{2}}\right)$ with a regularity $H^{2}(\Omega)$ were established in [14] without additional assumptions on the contact/friction set. This result has been improved recently in [24] and optimal rates have been recovered if the penalty parameter is large enough. An important step forward for the discretization of contact problems was accomplished in [13] by combining Nitsche's method with FEM. The FEM-Nitsche method differs from standard penalty techniques which are generally not consistent. Moreover no additional unknown (Lagrange multiplier) is needed and therefore no discrete inf-sup condition must be fulfilled contrary to mixed methods. For contact problems with Tresca friction discretized with FEM-Nitsche, optimal energy-error convergence of order $O\left(h^{r}\right)$ has been proved in [11] with the regularity $H^{1+r}(\Omega), r \in\left(\frac{1}{2}, k\right]$, where $k \geq 1$ is the polynomial degree of the Lagrange finite elements. To this purpose there is no need of any additional assumption on the contact/friction set. Note that the technical difficulties associated with the treatment of contact and friction condition when Nitsche's technique is not employed, are not limited to FEM, but appear as well for other discretizations such as discontinuous Galerkin (DG) [32] or Virtual finite elements (VEM) [33].

The devising and analysis of HHO-Nitsche methods was started in [10] for the scalar Signorini problem. Therein a face version and a cell version were analyzed, depending on the choice of the discrete unknown used to formulate the penalty terms. The cell version used cell unknowns of order $(k+1)$ (these unknowns can be eliminated by static condensation) and a modified reconstruction operator inspired from the unfitted HHO method from [9], leading to energy error estimates of order $\mathcal{O}\left(h^{r}\right)$ with $H^{1+r}$-regularity, $r \in\left(\frac{1}{2}, k+\right.$ 1]. Unfortunately, the modification of the reconstruction operator is not convenient in the context of elasticity as it hampers a key commuting property with the divergence operator which is crucial in the incompressible limit. This difficulty is circumvented in the present work by using face polynomials of order $(k+1)$ on the faces located on the contact/friction boundary. The numerical analysis also involves two novelties. Firstly, the error analysis, which adapts ideas from [15, 16] for FEM-Nitsche to HHONitsche, is more involved than [10] since it covers all the symmetry variants and since it hinges on a sharper bound on the consistency error allowing for a sharper threshold on the penalty parameters, especially in the case of the skew-symmetric variant. Secondly, for the first time concerning FEM-Nitsche as well, we track explicitly the dependency of the penalty parameters on the Lamé parameters for the various symmetry variants. Furthermore the present study is completed with 2D and 3D numerical tests including 


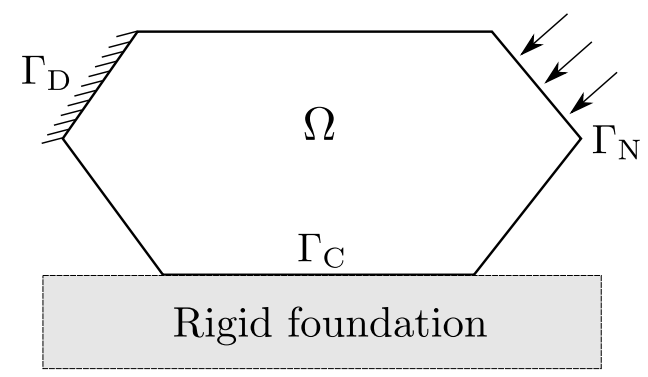

Figure 1: Geometry of the contact problem.

a prototype for an industrial application. Finally, let us mention that polyhedral discretizations for contact and friction problems have received some attention recently, as motivated by some numerical evidence illustrating their flexibility and accuracy. These discretizations use for instance VEM [35, 33], the weak Galerkin (WG) method [28] or the hybridizable discontinous Galerkin (HDG) methods [36], combined with different techniques to handle contact and friction (such as a direct approximation of the variational equality, node-to-node contact, penalty, Lagrange multipliers). The present work constitutes, to our knowledge, the first polyhedral discretization method for frictional contact problems using Nitsche's technique.

This paper is organized as follows. The model problem is described in Section 2. The HHO-Nitsche method is introduced in Section 3, and the stability and error analysis is contained in Section 4 . Numerical results are discussed in Section 5.

\section{Model problem}

Let $\Omega$ be a polygon/polyhedron in $\mathbb{R}^{d}, d \in\{2,3\}$, representing the reference configuration of an elastic body. The boundary $\partial \Omega$ is partitioned into three nonoverlapping parts (see Fig 1): the Dirichlet boundary $\Gamma_{\mathrm{D}}$, the Neumann boundary $\Gamma_{\mathrm{N}}$, and the contact/friction boundary $\Gamma_{\mathrm{C}}$. We assume meas $\left(\Gamma_{\mathrm{D}}\right)>0$ to prevent rigid body motions and meas $\left(\Gamma_{\mathrm{C}}\right)>0$ to ensure that contact is present. The small strain assumption is made, as well as plane strain if $d=2$. The linearized strain tensor associated with a displacement field $\underline{v}: \Omega \rightarrow \mathbb{R}^{d}$ is $\varepsilon(\underline{v}):=\frac{1}{2}\left(\boldsymbol{\nabla} \underline{v}+\boldsymbol{\nabla} \underline{v}^{T}\right) \in \mathbb{R}_{\mathrm{sym}}^{d \times d}$. Assuming isotropic behavior, the Cauchy stress tensor resulting from the strain tensor $\varepsilon(\underline{v})$ is denoted by $\boldsymbol{\sigma}(\underline{v})$ and is given by

$$
\boldsymbol{\sigma}(\underline{v})=2 \mu \varepsilon(\underline{v})+\lambda \operatorname{trace}(\varepsilon(\underline{v})) \boldsymbol{I}_{d} \in \mathbb{R}_{\mathrm{sym}}^{d \times d},
$$

where $\mu$ and $\lambda$ are the Lamé coefficients of the material satisfying $\mu>0$ and $3 \lambda+2 \mu>0$, and $\boldsymbol{I}_{d}$ is the identity tensor of order $d$. In what follows, we set $\kappa:=\max \left(1, \frac{\lambda}{2 \mu}\right)$. Let $\underline{n}$ be the unit outward normal vector to $\Omega$. On the boundary we consider the following decompositions into normal and tangential components:

$$
\underline{v}=v_{n} \underline{n}+\underline{v}_{t} \quad \text { and } \quad \underline{\sigma}_{n}(\underline{v}):=\boldsymbol{\sigma}(\underline{v}) \cdot \underline{n}=\sigma_{n}(\underline{v}) \underline{n}+\underline{\sigma}_{t}(\underline{v})
$$

where $v_{n}:=\underline{v} \cdot \underline{n}$ and $\sigma_{n}(\underline{v}):=\underline{\sigma}_{n}(\underline{v}) \cdot \underline{n}$ (so that $\underline{v}_{t} \cdot \underline{n}=0$ and $\left.\underline{\sigma}_{t}(\underline{v}) \cdot \underline{n}=0\right)$.

The body is subjected to volume forces $f \in L^{2}\left(\Omega ; \mathbb{R}^{d}\right)$ in $\Omega$ and to surface loads $g_{\mathrm{N}} \in L^{2}\left(\Gamma_{\mathrm{N}} ; \mathbb{R}^{d}\right)$ on $\Gamma_{\mathrm{N}}$, and it is clamped on $\Gamma_{\mathrm{D}}$ (for simplicity). The model problem consists in finding the displacement field $\underline{u}: \Omega \rightarrow \mathbb{R}^{d}$ such that

$$
\begin{cases}\underline{\nabla} \cdot \boldsymbol{\sigma}(\underline{u})+\underline{f}=\underline{0} & \text { in } \Omega, \\ \underline{u}=\underline{0} & \text { on } \Gamma_{\mathrm{D}} \\ \underline{\sigma}_{n}(\underline{u})=\underline{g}_{\mathrm{N}} & \text { on } \Gamma_{\mathrm{N}}, \\ (3) \text { and }(4) \text { hold true } & \text { on } \Gamma_{\mathrm{C}},\end{cases}
$$

where the unilateral contact conditions on $\Gamma_{\mathrm{C}}$ are as follows:

$$
\text { (i) } u_{n} \leq 0, \quad \text { (ii) } \sigma_{n}(\underline{u}) \leq 0, \quad \text { (iii) } \sigma_{n}(\underline{u}) u_{n}=0,
$$


whereas the Tresca friction conditions on $\Gamma_{\mathrm{C}}$ read (see, e.g., [30, Equ. (10.8)]):

$$
\text { (iv) }\left|\underline{\sigma}_{t}(\underline{u})\right| \leq s \quad \text { if } \underline{u}_{t}=\underline{0}, \quad\left(\text { v) } \underline{\sigma}_{t}(\underline{u})=-s \frac{\underline{u}_{t}}{\left|\underline{u}_{t}\right|} \quad \text { if }\left|\underline{u}_{t}\right|>0,\right.
$$

where $s \geq 0$ is a given threshold and $|\cdot|$ stands for the Euclidean norm in $\mathbb{R}^{d}$ (or the absolute value depending on the context). More generally $s$ can be a nonnegative-valued function on $\Gamma_{\mathrm{C}}$.

Remark 2.1 (Variants). The case of frictionless contact is recovered by setting $s:=0$ in (4). The case of bilateral contact with Tresca friction can be considered by keeping (4) whereas (3) is substituted by the following equation:

$$
u_{n}=0 \text { on } \Gamma_{\mathrm{C}} .
$$

In the case of unilateral contact with Tresca friction, nonzero tangential stress $\left(\left|\underline{\sigma}_{t}(\underline{u})\right|>0\right)$ can occur in regions with no-adhesion $\left(u_{n}<0\right)$, which is not expected physically (for Coulomb friction, $\underline{\sigma}_{t}(\underline{u})=0$ when $u_{n}<0$ ). The setting of bilateral contact prevents such situations (since $u_{n}=0$, there are no regions with no-adhesion). Therefore, the setting of bilateral contact with Tresca friction can be useful for the numerical simulation of specific situations that essentially correspond to persistent contact between elastic bodies with a high intensity of contact pressures (see, e.g., [30, Section 10.3] or [31]).

We introduce the Hilbert space $\underline{V}_{\mathrm{D}}$ and the convex cone $\underline{K}$ such that

$$
\underline{V}_{\mathrm{D}}:=\left\{\underline{v} \in H^{1}\left(\Omega ; \mathbb{R}^{d}\right) \mid \underline{v}=\underline{0} \text { on } \Gamma_{\mathrm{D}}\right\}, \quad \underline{K}:=\left\{\underline{v} \in \underline{V}_{\mathrm{D}} \mid v_{n} \leq 0 \text { on } \Gamma_{\mathrm{C}}\right\},
$$

i.e., the Dirichlet condition on $\Gamma_{\mathrm{D}}$ is explicitly enforced in the space $\underline{V}_{\mathrm{D}}$ and the non-interpenetration condition on $\Gamma_{\mathrm{C}}$ is explicitly enforced in the cone $\underline{K}$. We define the following bilinear form and the following linear and nonlinear forms:

$$
\begin{aligned}
a(\underline{v}, \underline{w}) & :=(\boldsymbol{\sigma}(\underline{v}), \boldsymbol{\varepsilon}(\underline{w}))_{\Omega}=2 \mu(\varepsilon(\underline{v}), \varepsilon(\underline{w}))_{\Omega}+\lambda(\nabla \cdot \underline{v}, \nabla \cdot \underline{w})_{\Omega}, \\
\ell(\underline{w}) & :=(\underline{f}, \underline{w})_{\Omega}+\left(\underline{g_{\mathrm{N}}}, \underline{w}\right)_{\Gamma_{\mathrm{N}}}, \quad j(\underline{w}):=\left(s,\left|\underline{w}_{t}\right|\right)_{\Gamma_{\mathrm{C}}},
\end{aligned}
$$

for any $\underline{v}$ and $\underline{w}$ in $\underline{V}_{\mathrm{D}}$, where the notation $(\cdot, \cdot)_{\omega}$ stands for the $L^{2}$-inner product on the set $\omega$. The weak formulation of (2) as a variational inequality of the second kind is

$$
\left\{\begin{array}{l}
\text { Find } \underline{u} \in \underline{K} \text { such that } \\
a(\underline{u}, \underline{w}-\underline{u})+j(\underline{w})-j(\underline{u}) \geq \ell(\underline{w}-\underline{u}), \quad \forall \underline{w} \in \underline{K} .
\end{array}\right.
$$

This problem admits a unique solution according, e.g., to [30, Theorem 10.2].

An important observation is that it is possible to reformulate the contact and friction conditions (3)-(4) as nonlinear equations. For any real number $x \in \mathbb{R}$, let $[x]_{\ominus}:=\min (x, 0)$ denote its projection onto the closed convex subset $\mathbb{R}^{-}:=(-\infty, 0]$. Moreover, let $[\cdot]_{\alpha}$ denote the orthogonal projection onto $\mathscr{B}(\underline{0}, \alpha) \subset \mathbb{R}^{d}$, where $\mathscr{B}(\underline{0}, \alpha)$ is the closed ball centered at the origin $\underline{0}$ and of radius $\alpha>0$, i.e., for all $\underline{x} \in \mathbb{R}^{d}$, we have $[\underline{x}]_{\alpha}:=\underline{x}$ if $|\underline{x}| \leq \alpha$ and $[\underline{x}]_{\alpha}:=\alpha \underline{\underline{x}}$ if $|\underline{x}|>\alpha$. The following result has been pointed out in [20] (see also [11] for formal proofs).

Proposition 2.2 (Reformulation as nonlinear conditions). Let $\gamma_{n}$ and $\gamma_{t}$ be positive functions on $\Gamma_{C}$. The conditions (3)-(4) enforcing unilateral contact with Tresca friction can be reformulated as follows:

$$
\begin{aligned}
\sigma_{n}(\underline{u}) & =\left[\tau_{n}(\underline{u})\right]_{\ominus}, & & \tau_{n}(\underline{u}):=\sigma_{n}(\underline{u})-\gamma_{n} u_{n}, \\
\underline{\sigma}_{t}(\underline{u}) & =\left[\underline{\tau}_{t}(\underline{u})\right]_{s}, & & \underline{\tau}_{t}(\underline{u}):=\underline{\sigma}_{t}(\underline{u})-\gamma_{t} \underline{u}_{t} .
\end{aligned}
$$

\section{HHO-Nitsche method}

In this section we devise and analyze the HHO-Nitsche method to approximate the frictional contact problem $(8)$. 


\subsection{Meshes and discrete unknowns}

Let $\left(\mathcal{T}_{h}\right)_{h>0}$ be a mesh sequence, where for all $h>0$, the mesh $\mathcal{T}_{h}$ is composed of nonempty disjoint cells such that $\bar{\Omega}=\bigcup_{T \in \mathcal{T}_{h}} \bar{T}$. The mesh cells are conventionally open subsets in $\mathbb{R}^{d}$ (not necessarily convex), and they can have a polygonal/polyhedral shape with straight edges (if $d=2$ ) or planar faces (if $d=3$ ). This setting in particular allows for meshes with hanging nodes. The mesh sequence $\left(\mathcal{T}_{h}\right)_{h>0}$ is assumed to be shape-regular in the sense of [22]. In a nutshell, each mesh $\mathcal{T}_{h}$ admits a matching simplicial submesh $\Im_{h}$ having locally equivalent length scales to those of $\mathcal{T}_{h}$, and the mesh sequence $\left(\Im_{h}\right)_{h>0}$ is shape-regular in the usual sense of Ciarlet. The meshsize is denoted $h:=\max _{T \in \mathcal{T}_{h}} h_{T}$, with $h_{T}$ the diameter of the cell $T$ and $\underline{n}_{T}$ denotes the unit outward normal to $T$. Discrete trace and inverse inequalities in the usual form are available on shape-regular polyhedral mesh sequences (see, e.g., [21]).

A closed subset $F$ of $\bar{\Omega}$ is called a mesh face if it is a subset with nonempty relative interior of some affine hyperplane $H_{F}$ and if (i) either there are two distinct mesh cells $T_{1}, T_{2} \in \mathcal{T}_{h}$ so that $F=\partial T_{1} \cap \partial T_{2} \cap H_{F}$ (and $F$ is called an interface) (ii) or there is one mesh cell $T_{1} \in \mathcal{T}_{h}$ so that $F=\partial T_{1} \cap \Gamma \cap H_{F}$ (and $F$ is called a boundary face). Remark that this definition allows for hanging nodes, since a mesh cell can have various faces that belong to the same hyperplane. The mesh faces are collected in the set $\mathcal{F}_{h}$ which is further partitioned into the subset of interfaces $\mathcal{F}_{h}^{\mathrm{i}}$ and the subset of boundary faces $\mathcal{F}_{h}^{\mathrm{b}}$. We assume that the meshes are compatible with the boundary partition $\partial \Omega=\overline{\Gamma_{\mathrm{D}}} \cup \overline{\Gamma_{\mathrm{N}}} \cup \overline{\Gamma_{\mathrm{C}}}$, which leads to the partition of the boundary faces as $\mathcal{F}_{h}^{\mathrm{b}}=\mathcal{F}_{h}^{\mathrm{b}, \mathrm{D}} \cup \mathcal{F}_{h}^{\mathrm{b}, \mathrm{N}} \cup \mathcal{F}_{h}^{\mathrm{b}, \mathrm{C}}$ (with obvious notation).

Let $k \geq 1$ be the polynomial degree. For all $T \in \mathcal{T}_{h}$, let $\mathcal{F}_{\partial T}$ be the collection of the mesh faces that are subsets of $\partial T$, let $\mathcal{F}_{\partial T}^{\mathrm{i}}:=\mathcal{F}_{\partial T} \cap \mathcal{F}_{h}^{\mathrm{i}}, \mathcal{F}_{\partial T}^{\mathrm{b}, \mathrm{C}}:=\mathcal{F}_{\partial T} \cap \mathcal{F}_{h}^{\mathrm{b}, \mathrm{C}}$, and we use a similar notation for $\mathcal{F}_{\partial T}^{\mathrm{b}, \mathrm{D}}$ and $\mathcal{F}_{\partial T}^{\mathrm{b}, \mathrm{N}}$. We set $\mathcal{F}_{\partial T}^{\backslash}:=\mathcal{F}_{\partial T}^{\mathrm{i}} \cup \mathcal{F}_{\partial T}^{\mathrm{b}, \mathrm{D}} \cup \mathcal{F}_{\partial T}^{\mathrm{b}, \mathrm{N}}$ for the collection of all the faces composing $\partial T$ except those located on $\Gamma_{\mathrm{C}}$. The local HHO discrete space is

$$
\widehat{U}_{T}^{k}:=\mathbb{P}^{k}\left(T ; \mathbb{R}^{d}\right) \times \mathbb{P}^{k / k+1}\left(\mathcal{F}_{\partial T} ; \mathbb{R}^{d}\right),
$$

where $\mathbb{P}^{k}\left(T ; \mathbb{R}^{d}\right)$ is composed of the restrictions to $T$ of $d$-variate polynomials of total degree at most $k$, and

$$
\mathbb{P}^{k / k+1}\left(\mathcal{F}_{\partial T} ; \mathbb{R}^{d}\right):=\mathbb{P}^{k}\left(\mathcal{F}_{\partial T}^{\backslash} ; \mathbb{R}^{d}\right) \times \mathbb{P}^{k+1}\left(\mathcal{F}_{\partial T}^{\mathrm{b}, \mathrm{C}} ; \mathbb{R}^{d}\right),
$$

where $\mathbb{P}^{k}\left(\mathcal{F}_{\partial T}^{\backslash} ; \mathbb{R}^{d}\right)$ and $\mathbb{P}^{k+1}\left(\mathcal{F}_{\partial T}^{\mathrm{b}, \mathrm{C}} ; \mathbb{R}^{d}\right)$ are composed of the restrictions to $\mathcal{F}_{\partial T}^{\backslash}$ and $\mathcal{F}_{\partial T}^{\mathrm{b}, \mathrm{C}}$, respectively, of piecewise $(d-1)$-variate polynomials of total degree at most $k$ and $(k+1)$, respectively. A generic element in $\underline{U}_{T}^{k}$ is a pair $\widehat{\underline{v}}_{T}:=\left(\underline{v}_{T}, \underline{v}_{\partial T}\right)$, where $\underline{v}_{T} \in \mathbb{P}^{k}\left(T ; \mathbb{R}^{d}\right)$ is the cell component and $\underline{v}_{\partial T} \in \mathbb{P}^{k / k+1}\left(\mathcal{F}_{\partial T} ; \mathbb{R}^{d}\right)$ is the face component. The degrees of freedom are illustrated in Fig. 2, where a dot indicates one degree of freedom (which is not necessarily computed as a point evaluation but can be for instance the moments against a selected set of basis functions defined on the cell or the face, see [17]).

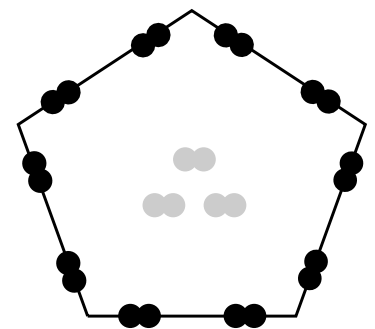

(a) Pentagonal cell with no contact face $\left(\mathcal{F}_{\partial T}^{\backslash}=\mathcal{F}_{\partial T}\right)$

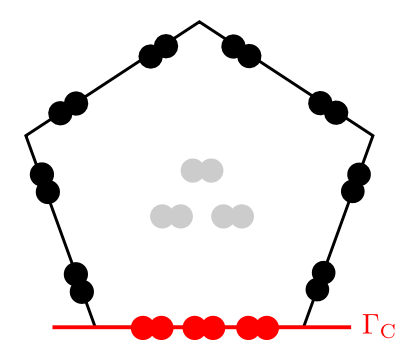

(b) Pentagonal cell with a contact face in red $\left(\mathcal{F}_{\partial T}^{\backslash} \varsubsetneqq \mathcal{F}_{\partial T}\right)$

Figure 2: Face (black or red) and cell (gray) degrees of freedom in $\widehat{U}_{T}^{k}$ for $k=1$ and $d=2$ (each dot represents a degree of freedom which is not necessarily a point evaluation).

Remark 3.1 (Degree on contact faces). We use a polynomial order $(k+1)$ on the faces located on the contact boundary (it is also possible to use this order on all the boundary faces). This choice is motivated by the error analysis, where it will be shown that it allows one to recover error bounds with optimal convergence order. Moreover this choice increases only marginally the computational cost with respect to using the same order $k$ for all the faces. 


\subsection{Local HHO operators}

The first key ingredient in the devising of the HHO method is a local symmetric strain reconstruction in each mesh cell $T \in \mathcal{T}_{h}$. Following [2,7,4], we define the local discrete symmetric gradient operator $\boldsymbol{E}: \widehat{U}_{T}^{k} \rightarrow \mathbb{P}^{k}\left(T ; \mathbb{R}_{\mathrm{sym}}^{d \times d}\right)$ such that, for all $\widehat{\widehat{v}}_{T} \in \underline{\underline{U}}_{T}^{k}, \boldsymbol{E}\left(\widehat{\widehat{v}}_{T}\right) \in \mathbb{P}^{k}\left(T ; \mathbb{R}_{\mathrm{sym}}^{d \times d}\right)$ solves the following local problem: For all $\boldsymbol{\tau} \in \mathbb{P}^{k}\left(T ; \mathbb{R}_{\mathrm{sym}}^{d \times d}\right)$,

$$
\left(\boldsymbol{E}\left(\underline{\widehat{v}}_{T}\right), \boldsymbol{\tau}\right)_{T}=-\left(\underline{v}_{T}, \underline{\nabla} \cdot \boldsymbol{\tau}\right)_{T}+\left(\underline{v}_{\partial T}, \boldsymbol{\tau} \cdot \underline{n}_{T}\right)_{\partial T} .
$$

The local problem (11) mimics an integration by parts at the discrete level. Moreover, the local discrete divergence operator $D: \widehat{U}_{T}^{k} \rightarrow \mathbb{P}^{k}(T ; \mathbb{R})$ is simply defined by taking the trace of the discrete symmetric gradient: For all $\underline{\widehat{v}}_{T} \in \underline{\widehat{U}}_{T}^{k}$,

$$
D\left(\underline{\underline{v}}_{T}\right):=\operatorname{trace}\left(\boldsymbol{E}\left(\widehat{\underline{v}}_{T}\right)\right) .
$$

The second key ingredient is a local stabilization operator $\underline{S}: \underline{U}_{T}^{k} \rightarrow \mathbb{P}^{k / k+1}\left(\partial T ; \mathbb{R}^{d}\right)$ used to penalize in a least-squares sense the difference between the face unknown $\underline{v}_{\partial T}$ and the trace of the cell unknown $\underline{v}_{T \mid \partial T}$. Let $\underline{\Pi}_{\partial T}^{k / k+1}$ and $\underline{\Pi}_{T}^{k}$ be the $L^{2}$-orthogonal projections onto $\mathbb{P}^{k / k+1}\left(\mathcal{F}_{\partial T} ; \mathbb{R}^{d}\right)$ and $\mathbb{P}^{k}\left(T ; \mathbb{R}^{d}\right)$, respectively. Then we set, for all $\underline{\widehat{v}}_{T} \in \underline{\widehat{U}}_{T}^{k}$,

$$
\underline{S}\left(\widehat{v}_{T}\right):=\underline{\Pi}_{\partial T}^{k / k+1}\left(\underline{v}_{\partial T}-\underline{R}\left(\underline{v}_{T}\right)_{\mid \partial T}\right)-\underline{\Pi}_{T}^{k}\left(\underline{v}_{T}-\underline{R}\left(\widehat{v}_{T}\right)\right)_{\mid \partial T} .
$$

Here $\underline{R}: \widehat{U}_{T}^{k} \rightarrow \mathbb{P}^{k+1}\left(T ; \mathbb{R}^{d}\right)$ is a local displacement reconstruction operator such that, for all $\widehat{\underline{v}}_{T} \in \underline{U}_{T}^{k}$, $\underline{R}\left(\widehat{\underline{v}}_{T}\right) \in \mathbb{P}^{k+1}\left(T ; \mathbb{R}^{d}\right)$ solves the following local problem: For all $\underline{w} \in \mathbb{P}^{k+1}\left(T ; \mathbb{R}^{d}\right)$,

$$
\left(\varepsilon\left(\underline{R}\left(\underline{\widehat{v}}_{T}\right)\right), \boldsymbol{\varepsilon}(\underline{w})\right)_{T}=\left(\varepsilon\left(\underline{v}_{T}\right), \boldsymbol{\varepsilon}(\underline{w})\right)_{T}+\left(\underline{v}_{\partial T}-\underline{v}_{T \mid \partial T}, \boldsymbol{\varepsilon}(\underline{w}) \cdot \underline{n}_{T}\right)_{\partial T} .
$$

The reconstructed displacement is uniquely defined by prescribing additionally that $\int_{T} \underline{R}\left(\underline{\underline{v}}_{T}\right) d T=\int_{T} \underline{v}_{T} d T$ and $\int_{T} \nabla^{s s}\left(\underline{R}\left(\underline{\widehat{v}}_{T}\right)\right) d T=\int_{\partial T} \frac{1}{2}\left(\underline{v}_{\partial T} \otimes \underline{n}_{T}-\underline{n}_{T} \otimes \underline{v}_{\partial T}\right) d \partial T$, where $\boldsymbol{\nabla}^{s s}(\underline{v}):=\frac{1}{2}\left(\boldsymbol{\nabla} \underline{v}-\nabla_{\underline{v}}{ }^{T}\right)$ is the skewsymmetric part of the gradient (see [22]). Comparing with (11), one readily sees that $\varepsilon\left(\underline{R}_{(}\left(\widehat{\underline{v}}_{T}\right)\right)$ is the $L^{2}$-orthogonal projection of $\boldsymbol{E}\left(\widehat{\underline{v}}_{T}\right)$ onto $\varepsilon\left(\mathbb{P}^{k+1}\left(T ; \mathbb{R}^{d}\right)\right)$.

We use the above operators to mimic locally the exact local bilinear form $a$ defined in (6) by means of the following local bilinear form defined on $\widehat{U}_{T}^{k} \times \underline{U}_{T}^{k}$ (compare with (6)):

$$
\begin{gathered}
\widehat{a}_{T}\left(\underline{\widehat{v}}_{T}, \underline{\widehat{w}}_{T}\right):=2 \mu\left(\boldsymbol{E}\left(\underline{\widehat{v}}_{T}\right), \boldsymbol{E}\left(\widehat{\widehat{w}}_{T}\right)\right)_{T}+\lambda\left(D\left(\underline{\widehat{v}}_{T}\right), D\left(\underline{\widehat{w}}_{T}\right)\right)_{T} \\
+2 \mu h_{T}^{-1}\left(\underline{S}\left(\underline{\widehat{v}}_{T}\right), \underline{S}\left(\underline{\widehat{w}}_{T}\right)\right)_{\partial T} .
\end{gathered}
$$

The stabilization term is weighted (as in the linear case) by the Lamé coefficient $\mu$.

\subsection{Global discrete problem}

For simplicity we employ the Nitsche technique only on the subset $\Gamma_{\mathrm{C}}$ where the nonlinear frictional contact conditions are enforced, whereas we resort to a strong enforcement of the homogeneous Dirichlet condition on the subset $\Gamma_{\mathrm{D}}$. The global discrete space for the HHO-Nitsche method is

$$
\widehat{\underline{U}}_{h}^{k}:=\mathbb{P}^{k}\left(\mathcal{T}_{h} ; \mathbb{R}^{d}\right) \times\left(\mathbb{P}^{k}\left(\mathcal{F}_{h}^{\mathrm{i}} \cup \mathcal{F}_{h}^{\mathrm{b}, \mathrm{D}} \cup \mathcal{F}_{h}^{\mathrm{b}, \mathrm{N}} ; \mathbb{R}^{d}\right) \times \mathbb{P}^{k+1}\left(\mathcal{F}_{h}^{\mathrm{b}, \mathrm{C}} ; \mathbb{R}^{d}\right)\right),
$$

leading to the notation $\underline{\underline{v}}_{h}:=\left(\left(\underline{v}_{T}\right)_{T \in \mathcal{T}_{h}},\left(\underline{v}_{F}\right)_{F \in \mathcal{F}_{h}}\right)$ for a generic element $\underline{\underline{v}}_{h} \in \underline{U}_{h}^{k}$. For all $T \in \mathcal{T}_{h}$, we denote by $\widehat{\widehat{v}}_{T}:=\left(\underline{v}_{T},\left(\underline{v}_{F}\right)_{F \in \mathcal{F}_{\partial T}}\right) \in \underline{U}_{T}^{k}$ the local components of $\widehat{\widehat{v}}_{h}$ attached to the mesh cell $T$ and the faces composing $\partial T$, and for any mesh face $F \in \mathcal{F}_{h}$, we denote by $\underline{v}_{F}$ the component of $\underline{\hat{v}}_{h}$ attached to the face $F$. We enforce strongly the homogeneous Dirichlet condition on $\Gamma_{\mathrm{D}}$ by considering the subspace

$$
\widehat{\widehat{U}}_{h, 0}^{k}:=\left\{\underline{\hat{v}}_{h} \in \underline{\widehat{U}}_{h}^{k} \mid \underline{v}_{F}=\underline{0} \quad \forall F \in \mathcal{F}_{h}^{\mathrm{b}, \mathrm{D}}\right\} .
$$

The HHO-Nitsche method uses a symmetry parameter $\theta \in\{-1,0,1\}$ and two penalty parameters $\gamma_{n}>0$ and $\gamma_{t}>0$ to enforce weakly the contact and friction conditions on $\Gamma_{\mathrm{C}}$, respectively. Choosing $\theta:=1$ leads to a symmetric formulation with a variational structure, choosing $\theta:=0$ is interesting to simplify the implementation by avoiding some terms in the formulation, and choosing $\theta:=-1$ allows one to improve on the stability of the method by exploiting its skew-symmetry and making it more robust in the incompressible 
limit. It is convenient to define the subset $\mathcal{T}_{h}^{\mathrm{C}}$ as the collection of the mesh cells having at least one boundary face on $\Gamma_{\mathrm{C}}$ and to set $\partial T^{\mathrm{C}}:=\partial T \cap \Gamma_{\mathrm{C}}$ for all $T \in \mathcal{T}_{h}^{\mathrm{C}}$. The subset $\mathcal{T}_{h}^{\mathrm{N}}$ is defined similarly, and we set $\partial T^{\mathrm{N}}:=\partial T \cap \Gamma_{\mathrm{N}}$ for all $T \in \mathcal{T}_{h}^{\mathrm{N}}$. We consider the following discrete HHO-Nitsche problem:

$$
\left\{\begin{array}{l}
\text { Find } \widehat{\widehat{u}}_{h} \in \widehat{\widehat{U}}_{h, 0}^{k} \text { such that } \\
\widehat{b}_{h}\left(\underline{\underline{\underline{x}}}_{h} ; \widehat{\widehat{w}}_{h}\right)=\widehat{\ell}_{h}\left(\widehat{\widehat{w}}_{h}\right) \quad \forall \underline{\widehat{w}}_{h} \in \underline{\widehat{U}}_{h, 0}^{k},
\end{array}\right.
$$

where the global discrete semilinear form $\widehat{b}_{h}$ and the global discrete linear form $\widehat{\ell}_{h}$ are defined as follows:

$$
\begin{aligned}
& \widehat{b}_{h}\left(\underline{\widehat{v}}_{h} ; \widehat{\widehat{w}}_{h}\right):=\sum_{T \in \mathcal{T}_{h}} \widehat{a}_{T}\left(\underline{\widehat{v}}_{T}, \widehat{\widehat{w}}_{T}\right) \\
& -\sum_{T \in \mathcal{T}_{h}^{\mathrm{C}}} \theta \frac{h_{T}}{\gamma_{n}}\left(\sigma_{n}\left(\widehat{\widehat{v}}_{T}\right), \sigma_{n}\left(\underline{\widehat{w}}_{T}\right)\right)_{\partial T^{\mathrm{C}}}-\sum_{T \in \mathcal{T}_{h}^{\mathrm{C}}} \theta \frac{h_{T}}{\gamma_{t}}\left(\underline{\sigma}_{t}\left(\widehat{\widehat{v}}_{T}\right), \underline{\sigma}_{t}\left(\widehat{\underline{w}}_{T}\right)\right)_{\partial T^{\mathrm{C}}} \\
& +\sum_{T \in \mathcal{T}_{h}^{\mathrm{C}}} \frac{h_{T}}{\gamma_{n}}\left(\left[\tau_{n}\left(\widehat{\underline{v}}_{T}\right)\right]_{\ominus},\left(\tau_{n}+(\theta-1) \sigma_{n}\right)\left(\underline{\widehat{w}}_{T}\right)\right)_{\partial T^{\mathrm{C}}} \\
& +\sum_{T \in \mathcal{T}_{h}^{\mathrm{C}}} \frac{h_{T}}{\gamma_{t}}\left(\left[\underline{\tau}_{t}\left(\widehat{\widehat{v}}_{T}\right)\right]_{s},\left(\underline{\tau}_{t}+(\theta-1) \underline{\sigma}_{t}\right)\left(\widehat{\underline{w}}_{T}\right)\right)_{\partial T^{\mathrm{C}}},
\end{aligned}
$$

and

$$
\widehat{\ell}_{h}\left(\widehat{\underline{w}}_{h}\right):=\sum_{T \in \mathcal{T}_{h}}\left(\underline{f}, \underline{w}_{T}\right)_{T}+\sum_{T \in \mathcal{T}_{h}^{\mathrm{N}}}\left(\underline{g}_{\mathrm{N}}, \underline{w}_{\partial T}\right)_{\partial T^{\mathrm{N}}} .
$$

Here, with a slight abuse of notation, we have written

$$
\boldsymbol{\sigma}\left(\underline{\widehat{w}}_{T}\right):=2 \mu \boldsymbol{E}\left(\underline{\widehat{\underline{w}}}_{T}\right)+\lambda D\left(\underline{\widehat{\underline{w}}}_{T}\right) \boldsymbol{I}_{d} \in \mathbb{P}^{k}\left(T ; \mathbb{R}_{\mathrm{sym}}^{d \times d}\right),
$$

together with the decomposition $\boldsymbol{\sigma}\left(\underline{\widehat{w}}_{T}\right) \cdot \underline{n}_{T}:=\sigma_{n}\left(\underline{\widehat{w}}_{T}\right) \underline{n}_{T}+\underline{\sigma}_{t}\left(\underline{\widehat{w}}_{T}\right)$, and we have introduced the linear operators (again with a slight abuse of notation)

$$
\tau_{n}\left(\underline{\underline{w}}_{T}\right):=\sigma_{n}\left(\underline{\widehat{w}}_{T}\right)-\frac{\gamma_{n}}{h_{T}} w_{\partial T, n}, \quad \underline{\tau}_{t}\left(\widehat{\underline{w}}_{T}\right):=\underline{\sigma}_{t}\left(\widehat{\underline{w}}_{T}\right)-\frac{\gamma_{t}}{h_{T}} \underline{w}_{\partial T, t},
$$

together with the decomposition $\underline{w}_{\partial T}:=w_{\partial T, n} \underline{n}_{T}+\underline{w}_{\partial T, t}$ for the face polynomials. Note that in the definition of $\tau_{n}$ and $\underline{\tau}_{t}$, the penalty parameters are rescaled by $h_{T}^{-1}$ in each mesh cell in $\mathcal{T}_{h}^{\mathrm{C}}$. Proposition 2.2 still holds true with this rescaling of the penalty parameters since the only requirement there is that $\gamma_{n}$ and $\gamma_{t}$ be positive.

Remark 3.2 (Comparison with FEM-Nitsche). In the FEM-Nitsche method devised in [11], one restricts the setting to simplicial meshes and considers the usual $\underline{H}^{1}$-conforming finite element space $\underline{V}_{h}$ composed of continuous functions that are piecewise polynomials of degree at most $k \geq 1$ in each mesh cell. The discrete problem is formulated in the subspace $\underline{V}_{h, 0}$ explicitly enforcing the Dirichlet condition on $\Gamma_{\mathrm{D}}$ and involves the global discrete semilinear form

$$
\begin{aligned}
b_{h}\left(\underline{v}_{h} ; \underline{w}_{h}\right):= & a\left(\underline{v}_{h}, \underline{w}_{h}\right)-\left(\frac{\theta}{\tilde{\gamma}} \sigma_{n}\left(\underline{v}_{h}\right), \sigma_{n}\left(\underline{w}_{h}\right)\right)_{\Gamma_{\mathrm{C}}}-\left(\frac{\theta}{\tilde{\gamma}} \underline{\sigma}_{t}\left(\underline{v}_{h}\right), \underline{\sigma}_{t}\left(\underline{w}_{h}\right)\right)_{\Gamma_{\mathrm{C}}} \\
& +\left(\frac{1}{\tilde{\gamma}}\left[\tau_{n}\left(\underline{v}_{h}\right)\right]_{\ominus},\left(\tau_{n}+(\theta-1) \sigma_{n}\right)\left(\underline{w}_{h}\right)\right)_{\Gamma_{\mathrm{C}}} \\
& +\left(\frac{1}{\tilde{\gamma}}\left[\underline{\tau}_{t}\left(\underline{v}_{h}\right)\right]_{s},\left(\underline{\tau}_{t}+(\theta-1) \underline{\sigma}_{t}\right)\left(\underline{w}_{h}\right)\right)_{\Gamma_{\mathrm{C}}},
\end{aligned}
$$

as well as the linear form $\ell_{h}\left(\underline{w}_{h}\right):=\ell\left(\underline{w}_{h}\right)$, where $a$ and $\ell$ are the same as for the continuous problem, the notation $\tau_{n}, \sigma_{n}, \underline{\tau}_{t}$, and $\underline{\sigma}_{t}$ is that employed for the exact solution, the symmetry parameter $\theta$ is taken again in $\{-1,0,1\}$, and the penalty parameter $\tilde{\gamma}$ is a piecewise constant function on $\Gamma_{\mathrm{C}}$ such that $\tilde{\gamma}_{\mid \partial T^{\mathrm{C}}}=h_{T}^{-1} \gamma$ with $\gamma>0$ for all $T \in \mathcal{T}_{h}^{\mathrm{C}}$. Note that there is only one penalty parameter in [11] since the analysis there did not consider the scaling with respect to the Lamé parameters.

Remark 3.3 (Comparison with [10]). There are various differences compared to the HHO-Nitsche method devised in [10] for the scalar Signorini problem. Here we address the vector-valued case and include Tresca friction. Moreover we use the face polynomials in the definition of the operators $\tau_{n}$ and $\underline{\tau}_{t}$ which corresponds to the face version considered in [10]. However we employ here a higher polynomial degree on those faces located on $\Gamma_{\mathrm{C}}$. 


\section{$4 \quad$ Stability and error analysis}

In this section we perform the stability and error analysis of the above HHO-Nitsche discretization of the frictional contact problem. We first collect some useful analysis tools. Then we establish a stability property and infer the well-posedness of the nonlinear discrete problem (17). The stability property is valid if the penalty parameters $\gamma_{n}$ and $\gamma_{t}$ are bounded from below, this bound being 0 for the skew-symmetric variant $\theta=-1$. Then we slightly tighten these minimal values to derive an error estimate bounding the energy error and the error on the nonlinear boundary condition on $\Gamma_{\mathrm{C}}$ and featuring optimal decay rates of order $(k+1)$ for smooth solutions. The following properties of projections onto a convex set will be useful:

$$
\begin{array}{ccc}
\left([x]_{\ominus}-[y]_{\ominus}\right)(x-y) \geq\left([x]_{\ominus}-[y]_{\ominus}\right)^{2} \geq 0, & \forall x, y \in \mathbb{R}, \\
\left([\underline{x}]_{s}-[\underline{y}]_{s}\right) \cdot(\underline{x}-\underline{y}) \geq\left|[\underline{x}]_{s}-[\underline{y}]_{s}\right|^{2} \geq 0, & \forall \underline{x}, \underline{y} \in \mathbb{R}^{d} .
\end{array}
$$

We use the symbol $C$ to denote a generic constant whose value can change at each occurrence as long as it is independent of the mesh size and the Lamé parameters. The value of $C$ can depend on the mesh regularity and the polynomial degree $k \geq 1$. We abbreviate as $a \lesssim b$ the inequality $a \leq C b$ with positive real numbers $a, b$ and a constant $C>0$ as above and whose value can change at each occurrence.

\subsection{Analysis tools for HHO operators}

We equip the space $\widehat{U}_{T}^{k}$ with the following local discrete strain seminorm:

$$
\left|\underline{\widehat{v}}_{T}\right|_{1, T}^{2}:=\left\|\varepsilon\left(\underline{v}_{T}\right)\right\|_{T}^{2}+h_{T}^{-1}\left\|\underline{v}_{\partial T}-\underline{v}_{T} \mid \partial T\right\|_{\partial T}^{2},
$$

where $\|\cdot\|_{T}$ (resp. $\|\cdot\|_{\partial T}$ and $\|\cdot\|_{\partial T^{\mathrm{C}}}$ ) is the $L^{2}$-norm on $T$ (resp. on $\partial T$ and $\partial T^{\mathrm{C}}$ ). Notice that $\left|\widehat{\hat{v}}_{T}\right|_{1, T}=0$ implies that $\underline{v}_{T}$ is a rigid-body motion and that $\underline{v}_{\partial T}$ is the trace of $\underline{v}_{T}$ on $\partial T$. The following local stability and boundedness properties of the strain reconstructon and stabilization operators are established as in [22, Lemma 4].

Lemma 4.1 (Boundedness and stability). Let $\boldsymbol{E}$ be defined by (11) and $\underline{S}$ by (13). There are $0<\alpha_{b}<$ $\alpha_{\sharp}<+\infty$ such that, for all $T \in \mathcal{T}_{h}$, all $h>0$, and all $\widehat{\widehat{v}}_{T} \in \underline{\widehat{U}}_{T}^{k}$, we have

$$
\alpha_{b}\left|\widehat{\underline{v}}_{T}\right|_{1, T} \leq\left(\left\|\boldsymbol{E}\left(\underline{\underline{v}}_{T}\right)\right\|_{T}^{2}+h_{T}^{-1}\left\|\underline{S}\left(\widehat{\underline{v}}_{T}\right)\right\|_{\partial T}^{2}\right)^{\frac{1}{2}} \leq \alpha_{\sharp}\left|\widehat{\underline{v}}_{T}\right|_{1, T} .
$$

The key operator in the HHO error analysis is the local interpolation operator $\widehat{I}_{T}^{k}: H^{1}\left(T ; \mathbb{R}^{d}\right) \rightarrow \underline{U}_{T}^{k}$ such that

$$
\widehat{I}_{T}^{k}(\underline{v}):=\left(\underline{\Pi}_{T}^{k}(\underline{v}), \underline{\Pi}_{\partial T}^{k / k+1}\left(\underline{v}_{\mid \partial T}\right)\right) \in \underline{U}_{T}^{k},
$$

for all $\underline{v} \in H^{1}\left(T ; \mathbb{R}^{d}\right)$ and all $T \in \mathcal{T}_{h}$. The global version $\underline{I}_{h}^{k}: \underline{V}_{\mathrm{D}} \rightarrow \underline{U}_{h, 0}^{k}$ is defined locally by setting the local HHO components of $\underline{I}_{h}^{k}(\underline{v})$ to $\left(\widehat{I}_{h}^{k}(\underline{v})\right)_{T}:=\underline{I}_{T}^{k}\left(\underline{v}_{\mid T}\right) \in \underline{U}_{T}^{k}$, for all $\underline{v} \in \underline{V}_{\mathrm{D}}$ and all $T \in \mathcal{T}_{h}$. This definition makes sense since the functions in $\underline{V}_{\mathrm{D}}$ do not jump across the mesh interfaces and vanish at the boundary faces located on $\Gamma_{\mathrm{D}}$. The HHO interpolation operator allows one to obtain important local commuting properties satisfied by the reconstruction operators (see, e.g., [22, Proposition 3]), namely we have

$$
\boldsymbol{E}\left(\widehat{I}_{T}^{k}(\underline{v})\right)=\Pi_{T}^{k}(\varepsilon(\underline{v})), \quad D\left(\underline{I}_{T}^{k}(\underline{v})\right)=\Pi_{T}^{k}(\nabla \cdot \underline{v}),
$$

for all $\underline{v} \in H^{1}\left(T ; \mathbb{R}^{d}\right)$, all $T \in \mathcal{T}_{h}$, and all $h>0$, where $\boldsymbol{\Pi}_{T}^{k}$ is the $L^{2}$-orthogonal projection onto $\mathbb{P}^{k}\left(T ; \mathbb{R}_{\mathrm{sym}}^{d \times d}\right)$ and $\Pi_{T}^{k}$ that onto $\mathbb{P}^{k}(T ; \mathbb{R})$. For all $\underline{v} \in H^{1+\nu}\left(\Omega ; \mathbb{R}^{d}\right), \nu>\frac{1}{2}$, and all $\underline{\widehat{w}}_{h} \in \underline{U}_{h, 0}^{k}$, let us set (the reason for the notation will become clear in the proof of Theorem 4.6 below)

$$
\mathbb{T}_{1,1}^{\prime}\left(\underline{v}, \underline{\underline{w}}_{h}\right):=\sum_{T \in \mathcal{T}_{h}}-\left(\left(\underline{\nabla} \cdot \boldsymbol{\sigma}(\underline{v}), \underline{w}_{T}\right)_{T}+\widehat{a}_{T}\left(\widehat{\underline{I}}_{T}^{k}(\underline{v}), \underline{\underline{w}}_{T}\right)\right)+\sum_{T \in \mathcal{T}_{h}^{\mathrm{N}} \cup \mathcal{T}_{h}^{\mathrm{C}}}\left(\underline{\sigma}_{n}(\underline{v}), \underline{w}_{\partial T}\right)_{\partial T^{\mathrm{N}} \cup \partial T^{\mathrm{C}}},
$$

where we recall that $\underline{\widehat{w}}_{T}=\left(\underline{w}_{T}, \underline{w}_{\partial T}\right)$ are the local components of the test function $\underline{\widehat{w}}_{h} \in \underline{\hat{U}}_{h, 0}^{k}$ attached to the mesh cell $T \in \mathcal{T}_{h}$. For a function $z \in H^{\nu}(T ; \mathbb{R}), \nu>\frac{1}{2}$, we employ the notation

$$
\|z\|_{\sharp, T}^{2}:=\|z\|_{T}^{2}+h_{T}\|z\|_{\partial T}^{2},
$$

and the same notation for tensor-valued functions. 
Lemma 4.2 (Consistency for linear elasticity). Let $\mathbb{T}_{1,1}^{\prime}\left(\underline{v}, \underline{\widehat{w}}_{h}\right)$ be defined in $(25)$ for all $\underline{v} \in H^{1+\nu}\left(\Omega ; \mathbb{R}^{d}\right)$, $\nu>\frac{1}{2}$, and all $\underline{\widehat{w}}_{h} \in \underline{\underline{U}}_{h, 0}^{k}$. The following holds true:

$$
\left|\mathbb{T}_{1,1}^{\prime}\left(\underline{v}, \widehat{\widehat{w}}_{h}\right)\right|^{2} \lesssim \mathbb{A}_{1}(\underline{v})\left(\sum_{T \in \mathcal{T}_{h}} 2 \mu\left|\underline{\widehat{w}}_{T}\right|_{1, T}^{2}\right),
$$

with the interpolation error

$$
\begin{aligned}
\mathbb{A}_{1}(\underline{v}):= & \sum_{T \in \mathcal{T}_{h}} \frac{1}{2 \mu}\left((2 \mu)^{2}\left\|\varepsilon(\underline{v})-\Pi_{T}^{k}(\varepsilon(\underline{v}))\right\|_{\sharp, T}^{2}+(2 \mu)^{2}\left\|\varepsilon\left(\underline{v}-\underline{\Pi}_{T}^{k+1}(\underline{v})\right)\right\|_{T}^{2}\right. \\
& \left.+\lambda^{2}\left\|\nabla \cdot \underline{v}-\Pi_{T}^{k}(\nabla \cdot \underline{v})\right\|_{\sharp, T}^{2}\right) .
\end{aligned}
$$

Proof. The proof essentially follows from [22, Theorem 8] and is only sketched here. Integrating by parts the term $\left(\underline{\nabla} \cdot \boldsymbol{\sigma}(\underline{v}), \underline{w}_{T}\right)_{T}$ for all $T \in \mathcal{T}_{h}$, using (1) together with the definitions (11)-(12) for $\boldsymbol{E}\left(\underline{\underline{w}}_{T}\right)$ and $D\left(\underline{\underline{w}}_{T}\right)$, respectively, re-arranging the terms, and setting $\boldsymbol{\eta}_{T}:=\varepsilon(\underline{v})-\boldsymbol{E}\left(\widehat{I}_{T}^{k}(\underline{v})\right)=\varepsilon(\underline{v})-\Pi_{T}^{k}(\varepsilon(\underline{v}))$ and $\zeta_{T}:=\nabla \cdot \underline{v}-D\left(\widehat{I}_{T}^{k}(\underline{v})\right)=\nabla \cdot \underline{v}-\Pi_{T}^{k}(\nabla \cdot \underline{v})$ (owing to the commuting properties (24)) leads to (details are skipped for brevity)

$$
\begin{aligned}
\mathbb{T}_{1,1}^{\prime}\left(\underline{v}, \underline{\underline{w}}_{h}\right)= & \sum_{T \in \mathcal{T}_{h}}\left(2 \mu\left(\boldsymbol{\eta}_{T}, \varepsilon\left(\underline{w}_{T}\right)\right)_{T}+2 \mu\left(\boldsymbol{\eta}_{T} \cdot \underline{n}_{T}, \underline{w}_{\partial T}-\underline{w}_{T}\right)_{\partial T}\right. \\
& -2 \mu h_{T}^{-1}\left(\underline{S}_{T}\left(\underline{I}_{T}^{k}(\underline{v})\right), \underline{S}\left(\widehat{\underline{w}}_{T}\right)\right)_{\partial T} \\
& \left.+\lambda\left(\zeta_{T}, \nabla \cdot \underline{w}_{T}\right)_{T}+\lambda\left(\zeta_{T} \underline{n}_{T}, \underline{w}_{\partial T}-\underline{w}_{T}\right)_{\partial T}\right) .
\end{aligned}
$$

Invoking the Cauchy-Schwarz inequality and the upper bound from Lemma 4.1 to estimate $h_{T}^{-\frac{1}{2}}\left\|\underline{S}\left(\underline{\widehat{w}}_{T}\right)\right\|_{\partial T}$, we infer that

$$
\begin{aligned}
\left|\mathbb{T}_{1,1}^{\prime}\left(\underline{v}, \underline{\widehat{w}}_{h}\right)\right|^{2} \lesssim & \left(\sum_{T \in \mathcal{T}_{h}} \frac{1}{2 \mu}\left((2 \mu)^{2}\left\|\boldsymbol{\eta}_{T}\right\|_{\sharp, T}^{2}+(2 \mu)^{2} h_{T}^{-1}\left\|\underline{S}\left(\widehat{I}_{T}^{k}(\underline{v})\right)\right\|_{\partial T}^{2}+\lambda^{2}\left\|\zeta_{T}\right\|_{\sharp, T}^{2}\right)\right) \\
& \times\left(\sum_{T \in \mathcal{T}_{h}} 2 \mu\left|\widehat{\underline{w}}_{T}\right|_{1, T}^{2}\right) .
\end{aligned}
$$

Finally combining the ideas used in [22, Eqs. $(20) \&(35)]$ with a local multiplicative trace inequality and a local Korn inequality, we infer that

$$
h_{T}^{-1}\left\|\underline{S}\left(\widehat{I}_{T}^{k}(\underline{v})\right)\right\|_{\partial T}^{2} \lesssim\left\|\varepsilon\left(\underline{v}-\underline{R}\left(\underline{I}_{T}^{k}(\underline{v})\right)\right)\right\|_{T}^{2} \leq\left\|\varepsilon\left(\underline{v}-\underline{\Pi}_{T}^{k+1}(\underline{v})\right)\right\|_{T}^{2},
$$

which completes the proof of $(26)$.

\subsection{Stability and well-posedness}

Let us first establish an important monotonicity property of the semilinear form $\widehat{b}_{h}$ under the assumption that the penalty parameters $\gamma_{n}$ and $\gamma_{t}$ are large enough. The lower bound on these parameters involves the constant $C_{\mathrm{dt}}$ from the following discrete trace inequality:

$$
\left\|v_{h}\right\|_{\partial T^{\mathrm{C}}} \leq C_{\mathrm{dt}} h_{T}^{-\frac{1}{2}}\left\|v_{h}\right\|_{T},
$$

for all $T \in \mathcal{T}_{h}^{\mathrm{C}}$, all $h>0$, and all $v_{h} \in \mathbb{P}^{k}\left(T ; \mathbb{R}^{q}\right), q \in\{1, d\}$. We equip the global HHO space $\widehat{\underline{U}}_{h, 0}^{k}$ with the norm

$$
\left\|\underline{\widehat{v}}_{h}\right\|_{\mu, \lambda}^{2}:=\sum_{T \in \mathcal{T}_{h}}\left(2 \mu\left|\widehat{\underline{v}}_{T}\right|_{1, T}^{2}+\lambda\left\|D\left(\widehat{\underline{v}}_{T}\right)\right\|_{T}^{2}\right) \text {. }
$$

That $\|\cdot\|_{\mu, \lambda}$ defines a norm on $\widehat{\underline{U}}_{h, 0}^{k}$ follows from the usual arguments since face components are null on all the faces in $\mathcal{F}_{h}^{\mathrm{b}, \mathrm{D}}$ and this set is nonempty by assumption. 
Lemma 4.3 (Monotonicity). Assume that the penalty parameters are such that

$$
\min \left(\kappa^{-1} \gamma_{n}, 2 \gamma_{t}\right) \geq 3(\theta+1)^{2} C_{\mathrm{dt}}^{2} \mu
$$

recalling that $\kappa:=\max \left(1, \frac{\lambda}{2 \mu}\right)$. Then the semilinear form $\widehat{b}_{h}$ is monotone and we have for all $\underline{\widehat{v}}_{h}, \underline{\widehat{w}}_{h} \in \underline{\widehat{U}}_{h, 0}^{k}$,

$$
\widehat{b}_{h}\left(\underline{\widehat{v}}_{h} ; \underline{\widehat{v}}_{h}-\underline{\widehat{w}}_{h}\right)-\widehat{b}_{h}\left(\underline{\widehat{w}}_{h} ; \underline{\widehat{v}}_{h}-\underline{\widehat{w}}_{h}\right) \geq \frac{1}{3} \min \left(1, \alpha_{b}^{2}\right)\left\|\underline{\widehat{v}}_{h}-\underline{\widehat{w}}_{h}\right\|_{\mu, \lambda}^{2} .
$$

Proof. Let $\underline{\widehat{v}}_{h}, \widehat{\widehat{w}}_{h} \in \underline{\widehat{U}}_{h, 0}^{k}$ and set $\underline{\widehat{z}}_{h}:=\underline{\widehat{v}}_{h}-\underline{\widehat{w}}_{h}$. Recalling the definition of the HHO-Nitsche semilinear form $\widehat{b}_{h}$ and exploiting the positivity of the local HHO bilinear form $\widehat{a}_{T}$, we infer that

$$
\sum_{T \in \mathcal{T}_{h}}\left(2 \mu\left(\left\|\boldsymbol{E}\left(\underline{\widehat{z}}_{T}\right)\right\|_{T}^{2}+h_{T}^{-1}\left\|\underline{S}\left(\underline{\widehat{z}}_{T}\right)\right\|_{\partial T}^{2}\right)+\lambda\left\|D\left(\underline{\widehat{z}}_{T}\right)\right\|_{T}^{2}\right) \leq \mathbb{T}_{1}+\mathbb{T}_{2, n}+\mathbb{T}_{2, t}-\mathbb{T}_{3, n}-\mathbb{T}_{3, t},
$$

where

$$
\begin{aligned}
\mathbb{T}_{1} & :=\widehat{b}_{h}\left(\underline{\widehat{v}}_{h} ; \widehat{\widehat{z}}_{h}\right)-\widehat{b}_{h}\left(\underline{\widehat{w}}_{h} ; \widehat{\widehat{z}}_{h}\right), \\
\mathbb{T}_{2, n} & :=\sum_{T \in \mathcal{T}_{h}^{\mathrm{C}}} \theta \frac{h_{T}}{\gamma_{n}}\left\|\sigma_{n}\left(\widehat{\widehat{z}}_{T}\right)\right\|_{\partial T^{\mathrm{C}}}^{2}, \quad \mathbb{T}_{2, t}:=\sum_{T \in \mathcal{T}_{h}^{\mathrm{C}}} \theta \frac{h_{T}}{\gamma_{t}}\left\|\underline{\sigma}_{t}\left(\widehat{\underline{z}}_{T}\right)\right\|_{\partial T^{\mathrm{C}}}^{2},
\end{aligned}
$$

and

$$
\begin{aligned}
\mathbb{T}_{3, n}:= & \sum_{T \in \mathcal{T}_{h}^{\mathrm{C}}} \frac{h_{T}}{\gamma_{n}}\left(\left[\tau_{n}\left(\widehat{\widehat{v}}_{T}\right)\right]_{\ominus}-\left[\tau_{n}\left(\underline{\widehat{w}}_{T}\right)\right]_{\ominus}, \tau_{n}\left(\widehat{\widehat{z}}_{T}\right)\right)_{\partial T^{\mathrm{C}}} \\
& +\sum_{T \in \mathcal{T}_{h}^{\mathrm{C}}}(\theta-1) \frac{h_{T}}{\gamma_{n}}\left(\left[\tau_{n}\left(\widehat{\widehat{v}}_{T}\right)\right]_{\ominus}-\left[\tau_{n}\left(\widehat{\widehat{w}}_{T}\right)\right]_{\ominus}, \sigma_{n}\left(\widehat{\underline{z}}_{T}\right)\right)_{\partial T^{\mathrm{C}}} \\
\mathbb{T}_{3, t}:= & \sum_{T \in \mathcal{T}_{h}^{\mathrm{C}}} \frac{h_{T}}{\gamma_{t}}\left(\left[\underline{\tau}_{t}\left(\underline{\hat{v}}_{T}\right)\right]_{s}-\left[\underline{\tau}_{t}\left(\underline{\widehat{w}}_{T}\right)\right]_{s}, \underline{\tau}_{t}\left(\underline{\underline{z}}_{T}\right)\right)_{\partial T^{\mathrm{C}}} \\
& +\sum_{T \in \mathcal{T}_{h}^{\mathrm{C}}}(\theta-1) \frac{h_{T}}{\gamma_{t}}\left(\left[\underline{\tau}_{t}\left(\widehat{\underline{v}}_{T}\right)\right]_{s}-\left[\underline{\tau}_{t}\left(\widehat{\underline{w}}_{T}\right)\right]_{s}, \underline{\sigma}_{t}\left(\widehat{\underline{z}}_{T}\right)\right)_{\partial T^{\mathrm{C}}} .
\end{aligned}
$$

Let us consider $\mathbb{T}_{2, n}-\mathbb{T}_{3, n}$. Setting $\delta_{T}:=\left[\tau_{n}\left(\widehat{\widehat{v}}_{T}\right)\right]_{\ominus}-\left[\tau_{n}\left(\underline{\widehat{w}}_{T}\right)\right]_{\ominus}$, we infer that

$$
\begin{aligned}
\mathbb{T}_{2, n}-\mathbb{T}_{3, n} & \leq \sum_{T \in \mathcal{T}_{h}^{\mathrm{C}}}\left(\theta \frac{h_{T}}{\gamma_{n}}\left\|\sigma_{n}\left(\widehat{\underline{z}}_{T}\right)\right\|_{\partial T^{\mathrm{C}}}^{2}-\left\|\delta_{T}\right\|_{\partial T^{\mathrm{C}}}^{2}-(\theta-1) \frac{h_{T}}{\gamma_{n}}\left(\delta_{T}, \sigma_{n}\left(\widehat{\underline{z}}_{T}\right)\right)_{\partial T^{\mathrm{C}}}\right) \\
& \leq \sum_{T \in \mathcal{T}_{h}^{\mathrm{C}}} \frac{1}{4}(\theta+1)^{2} \frac{h_{T}}{\gamma_{n}}\left\|\sigma_{n}\left(\widehat{\underline{z}}_{T}\right)\right\|_{\partial T^{\mathrm{C}}}^{2} \leq \sum_{T \in \mathcal{T}_{h}^{\mathrm{C}}} \frac{1}{4}(\theta+1)^{2} \frac{C_{\mathrm{dt}}^{2}}{\gamma_{n}}\left\|\sigma_{n}\left(\underline{z}_{T}\right)\right\|_{T}^{2}
\end{aligned}
$$

where we used (19) in the first bound, Young's inequality and the fact that $\theta+\frac{1}{4}(\theta-1)^{2}=\frac{1}{4}(\theta+1)^{2}$ in the second bound, and the discrete trace inequality (28) in the third bound. Recalling the definition (18) of the discrete HHO stress and using the triangle and Young's inequalities, we infer that

$$
\begin{aligned}
\mathbb{T}_{2, n}-\mathbb{T}_{3, n} & \leq \sum_{T \in \mathcal{T}_{h}^{\mathrm{C}}} \frac{1}{2}(\theta+1)^{2} \frac{C_{\mathrm{dt}}^{2}}{\gamma_{n}}\left((2 \mu)^{2}\left\|\boldsymbol{E}\left(\widehat{\underline{z}}_{T}\right)\right\|_{T}^{2}+\lambda^{2}\left\|D\left(\widehat{\underline{z}}_{T}\right)\right\|_{T}^{2}\right) \\
& \leq \sum_{T \in \mathcal{T}_{h}^{\mathrm{C}}}(\theta+1)^{2} \frac{C_{\mathrm{dt}}^{2}}{\gamma_{n}} \mu \kappa \times\left(2 \mu\left\|\boldsymbol{E}\left(\widehat{\underline{z}}_{T}\right)\right\|_{T}^{2}+\lambda\left\|D\left(\widehat{\underline{z}}_{T}\right)\right\|_{T}^{2}\right) .
\end{aligned}
$$

The reasoning to bound $\mathbb{T}_{2, t}-\mathbb{T}_{3, t}$ is similar except that $\underline{\sigma}_{t}\left(\underline{\underline{z}}_{T}\right)$ has only off-diagonal contributions and is therefore independent of $\lambda$. We infer that

$$
\mathbb{T}_{2, t}-\mathbb{T}_{3, t} \leq \sum_{T \in \mathcal{T}_{h}^{\mathrm{C}}} \frac{1}{2}(\theta+1)^{2} \frac{C_{\mathrm{dt}}^{2}}{\gamma_{t}} \mu \times 2 \mu\left\|\boldsymbol{E}\left(\widehat{\underline{z}}_{T}\right)\right\|_{T}^{2}
$$


Combining the above bounds and using the condition (29) leads to

$$
\mathbb{T}_{2, n}+\mathbb{T}_{2, t}-\mathbb{T}_{3, n}-\mathbb{T}_{3, t} \leq \sum_{T \in \mathcal{T}_{h}} \frac{2}{3}\left(2 \mu\left\|\boldsymbol{E}\left(\widehat{\underline{z}}_{T}\right)\right\|_{T}^{2}+\lambda\left\|D\left(\underline{\underline{z}}_{T}\right)\right\|_{T}^{2}\right) .
$$

Recalling (31) leads to

$$
\mathbb{T}_{1} \geq \sum_{T \in \mathcal{T}_{h}} \frac{1}{3}\left(2 \mu\left(\left\|\boldsymbol{E}\left(\underline{\underline{z}}_{T}\right)\right\|_{T}^{2}+h_{T}^{-1}\left\|\underline{S}\left(\widehat{\underline{z}}_{T}\right)\right\|_{\partial T}^{2}\right)+\lambda\left\|D\left(\widehat{\underline{z}}_{T}\right)\right\|_{T}^{2}\right) .
$$

Using the definition of $\mathbb{T}_{1}$, the lower bound from Lemma 4.1 , and the definition of the norm $\|\cdot\|_{\mu, \lambda}$ concludes the proof.

Using the argument from [8, Corollary 15, p. 126] (see [13] for the application to FEM-Nitsche), we infer from Lemma 4.3 the following well-posedness result.

Corollary 4.4 (Well-posedness). The discrete problem (17) is well-posed.

Remark 4.5 (Lower bound (29)). The monotonicity result stated in Lemma 4.3 is robust in the incompressible limit provided that the condition (29) does not imply that the penalty parameters $\gamma_{n}$ and $\gamma_{t}$ need to be chosen large when the ratio $\kappa$ is large (remember that this ratio becomes large in the incompressible limit). Robustness happens in the two following situations: 1) for the skew-symmetric variant $\theta=-1$, for which the penalty parameters $\gamma_{n}$ and $\gamma_{t}$ need only to be positive real numbers (instead, for $\theta \in\{0,1\}$, this property is lost for $\gamma_{n}$ which needs to scale as $\left.\mu \kappa\right) ; 2$ ) for bilateral contact and any value of $\theta$, since only the parameter $\gamma_{t}$ is used and it remains independent of $\kappa$.

\subsection{Error analysis}

This section contains our main theoretical results on the convergence of the HHO-Nitsche method for the frictional contact problem.

Theorem 4.6 (Error estimate). Let $\epsilon \in(0,1]$. Recall that $\kappa:=\max \left(1, \frac{\lambda}{2 \mu}\right)$. Assume that the penalty parameters are such that

$$
\min \left(\kappa^{-1} \gamma_{n}, 2 \gamma_{t}\right) \geq 3\left((\theta+1)^{2}+\epsilon\left(4+(\theta-1)^{2}\right)\right) C_{\mathrm{dt}}^{2} \mu
$$

Assume that the exact solution satisfies $\underline{u} \in H^{1+\nu}\left(\Omega ; \mathbb{R}^{d}\right), \nu>\frac{1}{2}$. Let $\widehat{\underline{u}}_{h}$ be the discrete solution of (17) with local components $\underline{\underline{u}}_{T}$ for all $T \in \mathcal{T}_{h}$. Then we have

$$
\begin{aligned}
& \sum_{T \in \mathcal{T}_{h}}\left(2 \mu\left\|\varepsilon(\underline{u})-\boldsymbol{E}\left(\underline{\widehat{u}}_{T}\right)\right\|_{T}^{2}+\lambda\left\|\nabla \cdot \underline{u}-D\left(\underline{\widehat{u}}_{T}\right)\right\|_{T}^{2}\right) \\
& +\frac{\epsilon}{2(1+\epsilon)} \sum_{T \in \mathcal{T}_{h}^{C}}\left(\frac{h_{T}}{\gamma_{n}}\left\|\left[\tau_{n}(\underline{u})\right]_{\ominus}-\left[\tau_{n}\left(\underline{\widehat{u}}_{T}\right)\right]_{\ominus}\right\|_{\partial T^{C}}^{2}+\frac{h_{T}}{\gamma_{t}}\left\|\left[\underline{\tau}_{t}(\underline{u})\right]_{s}-\left[\underline{\tau}_{t}\left(\underline{\widehat{u}}_{T}\right)\right]_{s}\right\|_{\partial T^{C}}^{2}\right) \\
& \lesssim \mathbb{A}_{1}(\underline{u})+\mathbb{A}_{2}(\underline{u}),
\end{aligned}
$$

with $\mathbb{A}_{1}(\underline{u})$ defined in $(27)$. Moreover $\mathbb{A}_{2}(\underline{u})$ is given by

$$
\begin{aligned}
\mathbb{A}_{2}(\underline{u}):= & \sum_{T \in \mathcal{T}_{h}^{C}} \frac{2}{\epsilon}\left(\frac{h_{T}}{\gamma_{n}}\left\|\delta \sigma_{n, T}\right\|_{\partial T^{C}}^{2}+\frac{\gamma_{n}}{h_{T}}\left\|\delta u_{n, T}\right\|_{\partial T^{C}}^{2}\right. \\
& \left.+\frac{h_{T}}{\gamma_{t}}\left\|\delta \underline{\sigma}_{t, T}\right\|_{\partial T^{C}}^{2}+\frac{\gamma_{t}}{h_{T}}\left\|\delta \underline{u}_{t, T}\right\|_{\partial T^{C}}^{2}\right),
\end{aligned}
$$

with $\delta \sigma_{n, T}:=\sigma_{n}(\underline{u})-\sigma_{n}\left(\widehat{I}_{T}^{k}(\underline{u})\right), \delta \underline{\sigma}_{t, T}:=\underline{\sigma}_{t}(\underline{u})-\underline{\sigma}_{t}\left(\widehat{I}_{T}^{k}(\underline{u})\right)$, the HHO interpolation operator is defined in $(23)$, and $\delta u_{n, T}$ and $\delta \underline{u}_{t, T}$ are the normal and tangential components on $\Gamma_{C}$ of $\delta \underline{u}_{T}:=\underline{u}_{\mid \partial T}-\underline{\Pi}_{\partial T}^{k+1}\left(\underline{u}_{\mid \partial T}\right)$. 
Proof. Let us set $\underline{\widehat{z}}_{h}:=\underline{\widehat{u}}_{h}-\underline{\underline{I}}_{h}^{k}(\underline{u})$, where $\underline{\underline{I}}_{h}^{k}: \underline{V}_{\mathrm{D}} \rightarrow \widehat{\underline{U}}_{h, 0}^{k}$ is the global HHO interpolation operator defined in Section 4.1. The same manipulations as in (31) lead to

$$
\sum_{T \in \mathcal{T}_{h}}\left(2 \mu\left(\left\|\boldsymbol{E}\left(\underline{\widehat{\underline{z}}}_{T}\right)\right\|_{T}^{2}+h_{T}^{-1}\left\|\underline{S}\left(\widehat{\widehat{\underline{z}}}_{T}\right)\right\|_{\partial T}^{2}\right)+\lambda\left\|D\left(\underline{\widehat{\underline{z}}}_{T}\right)\right\|_{T}^{2}\right) \leq \mathbb{T}_{1}+\mathbb{T}_{2, n}+\mathbb{T}_{2, t}-\mathbb{T}_{3, n}-\mathbb{T}_{3, t},
$$

where the terms on the right-hand side are defined as above by setting $\underline{\widehat{v}}_{h}:=\underline{\widehat{u}}_{h}$ and $\underline{\widehat{w}}_{h}:=\underline{\widehat{I}}_{h}^{k}(\underline{u})$. We use the fact that $\underline{\underline{u}}_{h}$ is the discrete solution to infer that $\mathbb{T}_{1}:=\widehat{b}_{h}\left(\widehat{\widehat{u}}_{h} ; \widehat{\widehat{z}}_{h}\right)-\widehat{b}_{h}\left(\underline{\widetilde{I}}_{h}^{k}(\underline{u}), \underline{\widehat{z}}_{h}\right)=\widehat{\widehat{\ell}}_{h}\left(\widehat{\widehat{z}}_{h}\right)-\widehat{b}_{h}\left(\underline{\widehat{I}}_{h}^{k}(\underline{u}), \underline{\widehat{z}}_{h}\right)$. Recalling the definition of $\widehat{b}_{h}$ and $\widehat{\ell}_{h}$, we obtain $\mathbb{T}_{1}:=\mathbb{T}_{1,1}+\mathbb{T}_{1,2, n}+\mathbb{T}_{1,2, t}-\mathbb{T}_{1,3, n}-\mathbb{T}_{1,3, t}$ with

$$
\begin{aligned}
\mathbb{T}_{1,1} & :=\sum_{T \in \mathcal{T}_{h}}\left(\left(\underline{f}, \underline{z}_{T}\right)_{T}-\widehat{a}_{T}\left(\widehat{\widehat{I}}_{T}^{k}(\underline{u}), \widehat{\underline{z}}_{T}\right)\right)+\sum_{T \in \mathcal{T}_{h}^{\mathrm{N}}}\left(\underline{g}_{\mathrm{N}}, \underline{z}_{\partial T}\right)_{\partial T^{\mathrm{N}}}, \\
\mathbb{T}_{1,2, n} & :=\sum_{T \in \mathcal{T}_{h}^{\mathrm{C}}} \theta \frac{h_{T}}{\gamma_{n}}\left(\sigma_{n}\left(\widehat{\underline{I}}_{T}^{k}(\underline{u})\right), \sigma_{n}\left(\underline{\widehat{\underline{z}}}_{T}\right)\right)_{\partial T^{\mathrm{C}}}, \\
\mathbb{T}_{1,3, n} & :=\sum_{T \in \mathcal{T}_{h}^{\mathrm{C}}} \frac{h_{T}}{\gamma_{n}}\left(\left[\tau_{n}\left(\widehat{\widehat{I}}_{T}^{k}(\underline{u})\right)\right]_{\ominus},\left(\tau_{n}+(\theta-1) \sigma_{n}\right)\left(\widehat{\underline{z}}_{T}\right)\right)_{\partial T^{\mathrm{C}}},
\end{aligned}
$$

and similar expressions for $\mathbb{T}_{1,2, t}$ and $\mathbb{T}_{1,3, t}$. We use the identities $\sigma_{n}\left(\widehat{\widehat{I}}_{T}^{k}(\underline{u})\right)=\left(\sigma_{n}\left(\underline{\widehat{I}}_{T}^{k}(\underline{u})\right)-\sigma_{n}(\underline{u})\right)+\sigma_{n}(\underline{u})$ and $\left[\tau_{n}\left(\widehat{\underline{I}}_{T}^{k}(\underline{u})\right)\right]_{\ominus}=\left(\left[\tau_{n}\left(\widehat{\underline{I}}_{T}^{k}(\underline{u})\right)\right]_{\ominus}-\left[\tau_{n}(\underline{u})\right]_{\ominus}\right)+\left[\tau_{n}(\underline{u})\right]_{\ominus}$ in $\mathbb{T}_{1,2, n}$ and $\mathbb{T}_{1,3, n}$, respectively, and obtain

$$
\mathbb{T}_{1}:=\mathbb{T}_{1,1}^{\prime}\left(\underline{u}, \underline{\widehat{\widehat{z}}}_{h}\right)-\mathbb{T}_{1,2, n}^{\prime}-\mathbb{T}_{1,2, t}^{\prime}+\mathbb{T}_{1,3, n}^{\prime}+\mathbb{T}_{1,3, t}^{\prime},
$$

where $\mathbb{T}_{1,1}^{\prime}\left(\underline{u}, \underline{\widehat{z}}_{h}\right)$ is defined in $(25)$ and (recall that $\left.\delta \sigma_{n, T}:=\sigma_{n}(\underline{u})-\sigma_{n}\left(\underline{\underline{I}}_{T}^{k}(\underline{u})\right)\right)$

$$
\begin{aligned}
\mathbb{T}_{1,2, n}^{\prime} & :=\sum_{T \in \mathcal{T}_{h}^{\mathrm{C}}} \theta \frac{h_{T}}{\gamma_{n}}\left(\delta \sigma_{n, T}, \sigma_{n}\left(\widehat{\underline{\underline{z}}}_{T}\right)\right)_{\partial T^{\mathrm{C}}}, \\
\mathbb{T}_{1,3, n}^{\prime} & :=\sum_{T \in \mathcal{T}_{h}^{\mathrm{C}}} \frac{h_{T}}{\gamma_{n}}\left(\left[\tau_{n}(\underline{u})\right]_{\ominus}-\left[\tau_{n}\left(\underline{\underline{I}}_{T}^{k}(\underline{u})\right)\right]_{\ominus},\left(\tau_{n}+(\theta-1) \sigma_{n}\right)\left(\widehat{\underline{z}}_{T}\right)\right)_{\partial T^{\mathrm{C}}},
\end{aligned}
$$

and similar expressions for $\mathbb{T}_{1,2, t}^{\prime}$ and $\mathbb{T}_{1,3, t}^{\prime}$. To make the term $\mathbb{T}_{1,1}^{\prime}\left(\underline{u}, \underline{\underline{z}}_{h}\right)$ appear in (35), we used that $\underline{\nabla} \cdot \boldsymbol{\sigma}(\underline{u})+\underline{f}=\underline{0}$ in $\Omega, \underline{\sigma}_{n}(\underline{u})=\underline{g}_{\mathrm{N}}$ on $\Gamma_{\mathrm{N}}$, whereas on $\Gamma_{\mathrm{C}}$ we used that $\sigma_{n}(\underline{u})=\left[\tau_{n}(\underline{u})\right]_{\ominus}, \frac{h_{T}}{\gamma_{n}}\left(\theta \sigma_{n}\left(\underline{\widehat{z}}_{T}\right)-\right.$ $\left.\left(\tau_{n}+(\theta-1) \sigma_{n}\right)\left(\underline{\widehat{z}}_{T}\right)\right)=z_{\partial T, n}$, a similar identity for the tangential component, and that $\underline{\sigma}_{n}(\underline{u}) \cdot \underline{z}_{\partial T}=$ $\sigma_{n}(\underline{u}) z_{\partial T, n}+\underline{\sigma}_{t}(\underline{u}) \cdot \underline{z}_{\partial T, t}$. Combining $\mathbb{T}_{2, n}$ with $\mathbb{T}_{1,2, n}^{\prime}$, we infer that

$$
\mathbb{T}_{2, n}^{\prime}:=\mathbb{T}_{2, n}-\mathbb{T}_{1,2, n}^{\prime}=\sum_{T \in \mathcal{T}_{h}^{\mathrm{C}}} \theta \frac{h_{T}}{\gamma_{n}}\left(\left\|\sigma_{n}\left(\widehat{\underline{z}}_{T}\right)\right\|_{\partial T^{\mathrm{C}}}^{2}-\left(\delta \sigma_{n, T}, \sigma_{n}\left(\widehat{\underline{z}}_{T}\right)\right)_{\partial T^{\mathrm{C}}}\right),
$$

together with a similar expression for $\mathbb{T}_{2, t}^{\prime}:=\mathbb{T}_{2, t}-\mathbb{T}_{1,2, t}^{\prime}$. Moreover, combining $\mathbb{T}_{3, n}$ with $\mathbb{T}_{1,3, n}^{\prime}$, we infer that

$$
\mathbb{T}_{3, n}^{\prime}:=-\mathbb{T}_{3, n}+\mathbb{T}_{1,3, n}^{\prime}=\sum_{T \in \mathcal{T}_{h}^{\mathrm{C}}} \frac{h_{T}}{\gamma_{n}}\left(\left[\tau_{n}(\underline{u})\right]_{\ominus}-\left[\tau_{n}\left(\widehat{\underline{u}}_{T}\right)\right]_{\ominus},\left(\tau_{n}+(\theta-1) \sigma_{n}\right)\left(\widehat{\underline{\underline{z}}}_{T}\right)\right)_{\partial T^{\mathrm{C}}},
$$

together with a similar expression for $\mathbb{T}_{3, t}^{\prime}:=-\mathbb{T}_{3, t}+\mathbb{T}_{1,3, t}^{\prime}$. Putting everything together, we infer that

$$
\begin{aligned}
& \sum_{T \in \mathcal{T}_{h}}\left(2 \mu\left(\left\|\boldsymbol{E}\left(\widehat{\underline{z}}_{T}\right)\right\|_{T}^{2}+h_{T}^{-1}\left\|\underline{S}\left(\widehat{\underline{z}}_{T}\right)\right\|_{\partial T}^{2}\right)+\lambda\left\|D\left(\underline{\underline{z}}_{T}\right)\right\|_{T}^{2}\right) \\
& \leq \mathbb{T}_{1,1}^{\prime}\left(\underline{u}, \widehat{\underline{z}}_{h}\right)+\mathbb{T}_{2, n}^{\prime}+\mathbb{T}_{2, t}^{\prime}+\mathbb{T}_{3, n}^{\prime}+\mathbb{T}_{3, t}^{\prime} .
\end{aligned}
$$

Let us now bound the terms $\left(\mathbb{T}_{2, n}^{\prime}+\mathbb{T}_{3, n}^{\prime}\right)$ and $\left(\mathbb{T}_{2, t}^{\prime}+\mathbb{T}_{3, t}^{\prime}\right)$. We only detail the bound on $\left(\mathbb{T}_{2, n}^{\prime}+\mathbb{T}_{3, n}^{\prime}\right)$ since the reasoning is similar for $\left(\mathbb{T}_{2, t}^{\prime}+\mathbb{T}_{3, t}^{\prime}\right)$. Recalling the above expression for $\mathbb{T}_{2, n}^{\prime}$ and using Young's inequality (with $\beta_{1}>0$ ) for the second term on the right-hand side, we infer that

$$
\mathbb{T}_{2, n}^{\prime} \leq \sum_{T \in \mathcal{T}_{h}^{\mathrm{C}}} \frac{h_{T}}{\gamma_{n}}\left(\left(\theta+\frac{\beta_{1}}{2}\right)\left\|\sigma_{n}\left(\widehat{\underline{z}}_{T}\right)\right\|_{\partial T^{\mathrm{C}}}^{2}+\frac{\theta^{2}}{2 \beta_{1}}\left\|\delta \sigma_{n, T}\right\|_{\partial T^{\mathrm{C}}}^{2}\right) .
$$


Turning to $\mathbb{T}_{3, n}^{\prime}$, since $\widehat{\widehat{\underline{z}}}_{T}=\underline{\widehat{u}}_{T}-\widehat{\underline{I}}_{T}^{k}(\underline{u})$ and the operator $\tau_{n}$ is linear, we have

$$
\left(\tau_{n}+(\theta-1) \sigma_{n}\right)\left(\underline{\widehat{\widehat{z}}}_{T}\right)=-\left(\tau_{n}(\underline{u})-\tau_{n}\left(\underline{\widehat{\underline{u}}}_{T}\right)\right)+\left(\tau_{n}(\underline{u})-\tau_{n}\left(\widehat{\underline{I}}_{T}^{k}(\underline{u})\right)\right)+(\theta-1) \sigma_{n}\left(\underline{\widehat{\underline{z}}}_{T}\right),
$$

so that we can re-arrange the terms composing $\mathbb{T}_{3, n}^{\prime}$ as follows:

$$
\begin{aligned}
\mathbb{T}_{3, n}^{\prime}= & \sum_{T \in \mathcal{T}_{h}^{\mathrm{C}}} \frac{h_{T}}{\gamma_{n}}\left(-\left(\left[\tau_{n}(\underline{u})\right]_{\ominus}-\left[\tau_{n}\left(\widehat{\widehat{u}}_{T}\right)\right]_{\ominus}, \tau_{n}(\underline{u})-\tau_{n}\left(\underline{\widehat{u}}_{T}\right)\right)_{\partial T^{\mathrm{C}}}\right. \\
& +\left(\left[\tau_{n}(\underline{u})\right]_{\ominus}-\left[\tau_{n}\left(\underline{\widehat{u}}_{T}\right)\right]_{\ominus}, \tau_{n}(\underline{u})-\tau_{n}\left(\widehat{\widehat{I}}_{T}^{k}(\underline{u})\right)\right)_{\partial T^{\mathrm{C}}} \\
& \left.+(\theta-1)\left(\left[\tau_{n}(\underline{u})\right]_{\ominus}-\left[\tau_{n}\left(\underline{\widehat{\underline{u}}}_{T}\right)\right]_{\ominus}, \sigma_{n}\left(\widehat{\widehat{\underline{z}}}_{T}\right)\right)_{\partial T^{\mathrm{C}}}\right) .
\end{aligned}
$$

Using (19) for the first term on the right-hand side and letting $\omega_{n, T}:=\left[\tau_{n}(\underline{u})\right]_{\ominus}-\left[\tau_{n}\left(\widehat{\underline{u}}_{T}\right)\right]_{\ominus}$ and $\delta \tau_{n, T}:=$ $\tau_{n}(\underline{u})-\tau_{n}\left(\widehat{\underline{I}}_{T}^{k}(\underline{u})\right)$, we infer that

$$
\mathbb{T}_{3, n}^{\prime} \leq \sum_{T \in \mathcal{T}_{h}^{\mathrm{C}}} \frac{h_{T}}{\gamma_{n}}\left(-\left\|\omega_{n, T}\right\|_{\partial T^{\mathrm{C}}}^{2}+\left(\omega_{n, T}, \delta \tau_{n, T}\right)_{\partial T^{\mathrm{C}}}+(\theta-1)\left(\omega_{n, T}, \sigma_{n}\left(\widehat{\underline{\underline{z}}}_{T}\right)\right)_{\partial T^{\mathrm{C}}}\right) .
$$

Using Young's inequality to bound the second and the third terms on the right-hand side (with $\beta_{2}>0$ and $\left.\beta_{3}>0\right)$, we infer that

$$
\mathbb{T}_{3, n}^{\prime} \leq \sum_{T \in \mathcal{T}_{h}^{\mathrm{C}}} \frac{h_{T}}{\gamma_{n}}\left(\left(-1+\frac{\beta_{2}}{2}+\frac{|\theta-1|}{2 \beta_{3}}\right)\left\|\omega_{n, T}\right\|_{\partial T^{\mathrm{C}}}^{2}+\frac{1}{2 \beta_{2}}\left\|\delta \tau_{n, T}\right\|_{\partial T^{\mathrm{C}}}^{2}+\frac{|\theta-1| \beta_{3}}{2}\left\|\sigma_{n}\left(\widehat{\underline{z}}_{T}\right)\right\|_{\partial T^{\mathrm{C}}}^{2}\right) .
$$

Putting the bounds on $\mathbb{T}_{2, n}^{\prime}$ and $\mathbb{T}_{3, n}^{\prime}$ together leads to

$$
\begin{aligned}
\mathbb{T}_{2, n}^{\prime}+\mathbb{T}_{3, n}^{\prime} \leq & \sum_{T \in \mathcal{T}_{h}^{\mathrm{C}}} \frac{h_{T}}{\gamma_{n}}\left(-\rho_{1}\left\|\omega_{n, T}\right\|_{\partial T^{\mathrm{C}}}^{2}+\rho_{2}\left\|\sigma_{n}\left(\widehat{\underline{z}}_{T}\right)\right\|_{\partial T^{\mathrm{C}}}^{2}\right. \\
& \left.+\frac{\theta^{2}}{2 \beta_{1}}\left\|\delta \sigma_{n, T}\right\|_{\partial T^{\mathrm{C}}}^{2}+\frac{1}{2 \beta_{2}}\left\|\delta \tau_{n, T}\right\|_{\partial T^{\mathrm{C}}}^{2}\right)
\end{aligned}
$$

with

$$
\rho_{1}:=1-\frac{\beta_{2}}{2}-\frac{|\theta-1|}{2 \beta_{3}}, \quad \rho_{2}:=\theta+\frac{\beta_{1}}{2}+\frac{|\theta-1| \beta_{3}}{2} .
$$

Let $\epsilon \in(0,1]$ and let us choose $\beta_{1}:=2 \epsilon, \beta_{2}:=\frac{\epsilon}{1+\epsilon}$, and $\beta_{3}:=\frac{|\theta-1|(1+\epsilon)}{2}$. Then we have $\rho_{1}=\frac{\epsilon}{2(1+\epsilon)}$ and $\rho_{2}=\frac{(\theta+1)^{2}}{4}+\epsilon\left(1+\frac{(\theta-1)^{2}}{4}\right)$, as well as $\frac{\theta^{2}}{2 \beta_{1}}=\frac{\theta^{2}}{4 \epsilon} \leq \frac{1}{4 \epsilon} \leq \frac{1}{\epsilon}$ and $\frac{1}{2 \beta_{2}}=\frac{1+\epsilon}{2 \epsilon} \leq \frac{1}{\epsilon}$. Using the above bound on $\mathbb{T}_{2, n}^{\prime}+\mathbb{T}_{3, n}^{\prime}$ together with a similar bound on $\mathbb{T}_{2, t}^{\prime}+\mathbb{T}_{3, t}^{\prime}$ in (36), we infer that

$$
\begin{aligned}
& \sum_{T \in \mathcal{T}_{h}}\left(2 \mu\left(\left\|\boldsymbol{E}\left(\underline{\underline{z}}_{T}\right)\right\|_{T}^{2}+h_{T}^{-1}\left\|\underline{S}\left(\underline{\underline{z}}_{T}\right)\right\|_{\partial T}^{2}\right)+\lambda\left\|D\left(\underline{\widehat{\underline{z}}}_{T}\right)\right\|_{T}^{2}\right) \\
& +\rho_{1} \sum_{T \in \mathcal{T}_{h}^{\mathrm{C}}}\left(\frac{h_{T}}{\gamma_{n}}\left\|\omega_{n, T}\right\|_{\partial T^{\mathrm{C}}}^{2}+\frac{h_{T}}{\gamma_{t}}\left\|\underline{\omega}_{t, T}\right\|_{\partial T^{\mathrm{C}}}^{2}\right) \\
& \leq \mathbb{T}_{1,1}^{\prime}\left(\underline{u}, \widehat{\widehat{z}}_{h}\right)+\sum_{T \in \mathcal{T}_{h}^{\mathrm{C}}} \rho_{2}\left(\frac{h_{T}}{\gamma_{n}}\left\|\sigma_{n}\left(\underline{\widehat{\underline{z}}}_{T}\right)\right\|_{\partial T^{\mathrm{C}}}^{2}+\frac{h_{T}}{\gamma_{t}}\left\|\underline{\sigma}_{t}\left(\underline{\widehat{\underline{z}}}_{T}\right)\right\|_{\partial T^{\mathrm{C}}}^{2}\right)+\mathbb{A}_{2}^{\prime}(\underline{u}),
\end{aligned}
$$

with $\underline{\omega}_{t, T}:=\left[\underline{\tau}_{t}(\underline{u})\right]_{s}-\left[\underline{\tau}_{t}\left(\underline{\widehat{u}}_{T}\right)\right]_{s}$ and

$$
\mathbb{A}_{2}^{\prime}(\underline{u}):=\sum_{T \in \mathcal{T}_{h}^{\mathrm{C}}} \frac{1}{\epsilon}\left(\frac{h_{T}}{\gamma_{n}}\left(\left\|\delta \sigma_{n, T}\right\|_{\partial T^{\mathrm{C}}}^{2}+\left\|\delta \tau_{n, T}\right\|_{\partial T^{\mathrm{C}}}^{2}\right)+\frac{h_{T}}{\gamma_{t}}\left(\left\|\delta \underline{\sigma}_{t, T}\right\|_{\partial T^{\mathrm{C}}}^{2}+\left\|\delta \underline{\tau}_{t, T}\right\|_{\partial T^{\mathrm{C}}}^{2}\right)\right),
$$

recalling that $\delta \sigma_{n, T}:=\sigma_{n}(\underline{u})-\sigma_{n}\left(\widehat{I}_{T}^{k}(\underline{u})\right), \delta \underline{\sigma}_{t, T}:=\underline{\sigma}_{t}(\underline{u})-\underline{\sigma}_{t}\left(\widehat{I}_{T}^{k}(\underline{u})\right)$ are defined below $(34), \delta \tau_{n, T}:=$ $\tau_{n}(\underline{u})-\tau_{n}\left(\widehat{\underline{I}}_{T}^{k}(\underline{u})\right)$ is defined above in this proof, and $\delta \underline{\tau}_{t, T}:=\underline{\tau}_{t}\left(\underline{u}^{\prime}\right)-\underline{\tau}_{t}\left(\widehat{I}_{T}^{k}(\underline{u})\right)$. Recalling the definitions of the operators $\tau_{n}$ and $\underline{\tau}_{t}$ and invoking the triangle and Young's inequalities, we infer that $\mathbb{A}_{2}^{\prime}(\underline{u}) \leq \mathbb{A}_{2}(\underline{u})$, 
with $\mathbb{A}_{2}(\underline{u})$ defined in $(34)$. Note importantly that the face component of $\widehat{I}_{T}^{k}(\underline{u})$ on $\partial T^{\mathrm{C}}$ is indeed $\underline{\Pi}_{\partial T}^{k+1}\left(\underline{u}_{\mid \partial T}\right)$ since the polynomial order is $(k+1)$ on the faces located on $\Gamma_{\mathrm{C}}$. Next, we absorb the traces of $\sigma_{n}\left(\underline{\widehat{z}}_{T}\right)$ and $\underline{\sigma}_{t}\left(\widehat{\underline{z}}_{T}\right)$ in (37) (in the term multiplied by $\rho_{2}$ ) by the positive terms from the left-hand side. To this purpose, we proceed as in the proof of Lemma 4.3, i.e., we invoke the discrete trace inequality (28) and the lower bound (32) on the penalty parameters. This yields

$$
\begin{aligned}
& \sum_{T \in \mathcal{T}_{h}} \frac{1}{3}\left(2 \mu\left(\left\|\boldsymbol{E}\left(\underline{\widehat{z}}_{T}\right)\right\|_{T}^{2}+h_{T}^{-1}\left\|\underline{S}\left(\widehat{\widehat{z}}_{T}\right)\right\|_{\partial T}^{2}\right)+\lambda\left\|D\left(\underline{\underline{z}}_{T}\right)\right\|_{T}^{2}\right) \\
& +\frac{\epsilon}{2(1+\epsilon)} \sum_{T \in \mathcal{T}_{h}^{\mathrm{C}}}\left(\frac{h_{T}}{\gamma_{n}}\left\|\omega_{n, T}\right\|_{\partial T^{\mathrm{C}}}^{2}+\frac{h_{T}}{\gamma_{t}}\left\|\underline{\omega}_{t, T}\right\|_{\partial T^{\mathrm{C}}}^{2}\right) \leq \mathbb{T}_{1,1}^{\prime}\left(\underline{u}, \underline{\widehat{z}}_{h}\right)+\mathbb{A}_{2}(\underline{u}) .
\end{aligned}
$$

Finally we invoke Lemma 4.2 and infer that $\left|\mathbb{T}_{1,1}^{\prime}\left(\underline{u}, \underline{\widehat{z}}_{h}\right)\right|^{2} \lesssim \mathbb{A}_{1}(\underline{u}) \sum_{T \in \mathcal{T}_{h}} 2 \mu\left|\underline{\underline{z}}_{T}\right|_{1, T}^{2}$. Owing to the lower bound from Lemma 4.1 and Young's inequality, we can hide the factor $\sum_{T \in \mathcal{T}_{h}} 2 \mu\left|\underline{\underline{z}}_{T}\right|_{1, T}^{2}$ on the left-hand side of the above inequality. We conclude the proof by means of a triangle inequality.

Remark 4.7 (Lower bound (32)). The minimal value of the penalty parameters from the lower bound in (32) is slightly tighter than that from the lower bound (29) and tends to it as $\epsilon \downarrow 0$. Formally one recovers the arguments from the proof of Lemma 4.3 (which invole only the two functions $\underline{\underline{v}}_{h}, \underline{\widehat{w}}_{h}$ instead of the three functions $\underline{\widehat{u}}_{h}, \underline{I}_{h}^{k}(\underline{u})$ and $\underline{u}$ as in the proof of Theorem 4.6) by setting $\epsilon:=0$ so that $\beta_{1}=\beta_{2}=0, \beta_{3}=\frac{|\theta-1|}{2}$, $\rho_{1}=0$ and $\rho_{2}=\frac{(\theta+1)^{2}}{4}$.

Convergence rates for smooth solutions can be inferred from Theorem 4.6 by using the approximation properties of the $L^{2}$-orthogonal projection on shape-regular polyhedral mesh sequences. Referring, e.g., to $[21,27]$ for proofs, we have

$$
\begin{aligned}
\left\|v-\Pi_{T}^{k+1}(v)\right\|_{T}+h_{T}^{\frac{1}{2}}\left\|v-\Pi_{T}^{k+1}(v)\right\|_{\partial T}+h_{T}\left\|\underline{\nabla}\left(v-\Pi_{T}^{k+1}(v)\right)\right\|_{T} & \\
& +h_{T}^{\frac{3}{2}}\left\|\underline{\nabla}\left(v-\Pi_{T}^{k+1}(v)\right)\right\|_{\partial T} \lesssim h_{T}^{1+r}|v|_{H^{1+r}(T)}
\end{aligned}
$$

for all $v \in H^{1+\nu}(T ; \mathbb{R}), \nu \in\left(\frac{1}{2}, k+1\right]$, all $T \in \mathcal{T}_{h}$, and all $h>0$. Similar bounds are available for the projection of vector-valued functions. Using (38) to bound $\mathbb{A}_{1}(\underline{u})$ and $\mathbb{A}_{2}(\underline{u})$ in (33) readily leads to the following error estimate (note that one can assume $\gamma_{t} \leq \gamma_{n}$ without loss of generality).

Corollary 4.8 ( $H^{1}$-error estimate). Keep the assumptions and notation from Theorem 4.6. Assume that the exact solution satisfies $\underline{u} \in H^{1+\nu}\left(\Omega ; \mathbb{R}^{d}\right)$ and $\nabla \cdot \underline{u} \in H^{\nu}(\Omega ; \mathbb{R}), \nu \in\left(\frac{1}{2}, k+1\right]$. Then we have

$$
\begin{aligned}
& \sum_{T \in \mathcal{T}_{h}}\left(2 \mu\left\|\varepsilon(\underline{u})-\boldsymbol{E}\left(\underline{\widehat{u}}_{T}\right)\right\|_{T}^{2}+\lambda\left\|\nabla \cdot \underline{u}-D\left(\underline{\widehat{u}}_{T}\right)\right\|_{T}^{2}\right) \\
& \quad+\frac{\epsilon}{2(1+\epsilon)} \sum_{T \in \mathcal{T}_{h}^{C}}\left(\frac{h_{T}}{\gamma_{n}}\left\|\left[\tau_{n}(\underline{u})\right]_{\ominus}-\left[\tau_{n}\left(\underline{\underline{u}}_{T}\right)\right]_{\ominus}\right\|_{\partial T^{C}}^{2}+\frac{h_{T}}{\gamma_{t}}\left\|\left[\underline{\tau}_{t}(\underline{u})\right]_{s}-\left[\underline{\tau}_{t}\left(\underline{\underline{u}}_{T}\right)\right]_{s}\right\|_{\partial T^{C}}^{2}\right) \\
& \lesssim \sum_{T \in \mathcal{T}_{h}}\left(\left(2 \mu+\frac{1}{\epsilon}\left(\frac{\mu^{2} \kappa^{2}}{\gamma_{n}}+\frac{\mu^{2}}{\gamma_{t}}+\gamma_{n}\right)\right) h_{T}^{2 r}|\underline{u}|_{H^{1+r}(T)}^{2}+\frac{\lambda^{2}}{2 \mu} h_{T}^{2 r}|\nabla \cdot \underline{u}|_{H^{r}(T)}^{2}\right) .
\end{aligned}
$$

Remark 4.9 (Choice of $\epsilon$, robustness). For $\theta \in\{0,1\}$, the value chosen for $\epsilon$ is not really important, and one can simply set $\epsilon=1$. Then (32) shows that $\gamma_{n} \sim \mu \kappa, \gamma_{t} \sim \mu$, and taking the square root in (39) shows that the error upper bound scales as $\kappa^{\frac{1}{2}}$. Instead, for $\theta=-1$, choosing $\epsilon$ arbitrarily small allows the lower bound on the parameters $\kappa^{-1} \gamma_{n}$ and $\gamma_{t}$ to be arbitrarily small as well, as is expected for the skew-symmetric variant. Note however that the choice of $\epsilon$ affects the error upper bound (39) which scales as $\epsilon^{-1}$ (a similar issue is also observed for Nitsche-FEM in [15]). A first possibility is to set $\epsilon \approx \kappa^{-1}$ leading to $\gamma_{n} \sim \mu$ and $\gamma_{t} \sim \mu \kappa^{-1}$, whereby the error estimate (39) delivers an upper bound scaling as $\kappa^{\frac{3}{2}}$ after taking the square root. A second possibility is to set $\epsilon=1$ and to take $\gamma_{n} \sim \mu \kappa$ to moderate the dependency on $\kappa$ of the upper bound in (39) which then scales as $\kappa^{\frac{1}{2}}$ after taking the square root.

Remark 4.10 (Choice of $k$ ). A usual smoothness assumption is $\underline{u} \in H^{\frac{5}{2}-\varepsilon}\left(\Omega ; \mathbb{R}^{d}\right), \varepsilon>0$, i.e., $\nu=\frac{3}{2}-\varepsilon$, as is generally the case when there is a transition between contact and no-contact. Then the maximal convergence rate is $\mathcal{O}\left(h^{\frac{3}{2}-\varepsilon}\right)$ and is reached for $k=1$. 


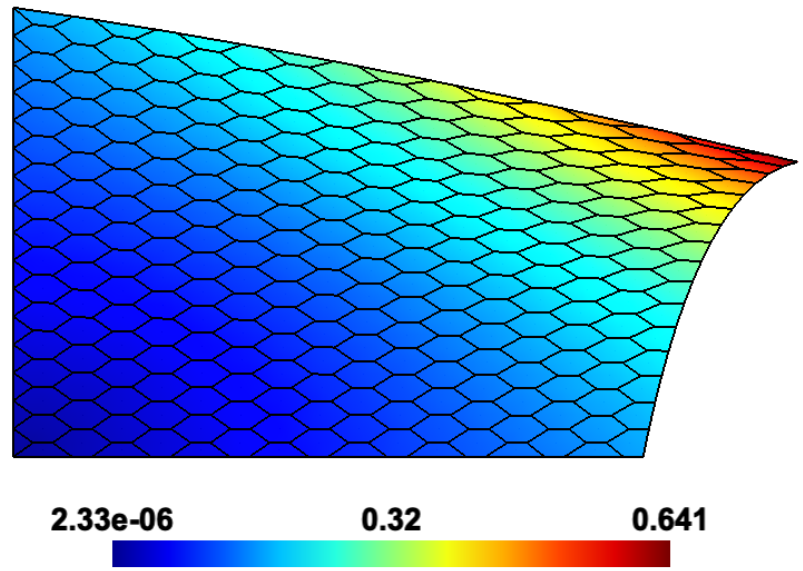

Figure 3: 2D manufactured solution: displacement Euclidean norm on the deformed configuration for $\mathrm{HHO}(1)$ and $\theta=0$ for a hexagonal mesh $(h=4.60 \mathrm{e}-2)$ (the contact boundary $\Gamma_{\mathrm{C}}$ is the bottom side).

Remark 4.11 (Face polynomials). Using face polynomials of order $(k+1)$ on the faces located on $\Gamma_{\mathrm{C}}$ is crucial to obtain the above error estimate in the optimal case where $\nu=k+1$. This allows us to invoke the approximation properties of $\underline{\Pi}_{\partial T}^{k+1}$ on $\partial T^{\mathrm{C}}$ when bounding $\mathbb{A}_{2}(\underline{u})$.

\section{$5 \quad$ Numerical experiments}

The goal of this section is to evaluate the proposed HHO-Nitsche method on two- and three-dimensional benchmarks: (i) a two-dimensional manufactured solution; (ii) a three-dimensional frictionless Hertz contact problem; (iii) a stick and slip transition, and (iv) a prototype for an industrial application. We employ the notation $\mathrm{HHO}(k)$ when using polynomials of order $k \geq 1$. The implementation of HHO methods is discussed in [17] and an open-source software is available ${ }^{1}$. The discrete nonlinear problem (17) is solved by a generalized Newton method as in [20]. In the present implementation, the penalty parameters for the stabilization and the friction/Tresca condition are proportional to $2 \mu$ and are scaled by the reciprocal of the diameter of the local face rather than the diameter of the local cell (these two length scales are uniformly equivalent owing to the shape-regularity of the mesh sequence). We compare our numerical results to the analytical solution whenever available or to numerical solutions obtained either from the literature or using the industrial open-source FEM software code_aster [26]. In this latter case we consider a mixed method called T2-LAC (see [1]) where the discrete unknowns are the piecewise quadratic displacement field and the piecewise constant contact pressure.

\subsection{D manufactured solution}

We consider the unit square $\Omega:=(0,1)^{2}$, we set $\Gamma_{\mathrm{C}}:=(0,1) \times\{0\}$ and $\Gamma_{\mathrm{D}}:=\{0,1\} \times(0,1) \cup(0,1) \times\{1\}$. The Lamé coefficients are $\mu:=2$ and $\lambda:=1000$ (which corresponds to a Poisson ratio of $\nu \simeq 0.499$ ). The manufactured solution is

$$
u_{x}(x, y):=\left(1+\frac{1}{1+\lambda}\right) x e^{x+y}, \quad u_{y}(x, y):=\left(-1+\frac{1}{1+\lambda}\right) y e^{x+y}
$$

The $x$-dependent friction threshold is $s(x):=\mu x^{2} \frac{\lambda+2}{6 \lambda+6}$. The displacement imposed on $\Gamma_{\mathrm{D}}$ is the trace of the manufactured solution, and the volume force is computed accordingly. The penalty parameters are taken as $\gamma_{n}=\gamma_{t}:=2 \mu$. In this test case, we consider hexagonal meshes to illustrate the polyhedral capabilities of the proposed HHO-Nitsche method. The displacement Euclidean norm of the manufactured solution is plotted in Fig. 3 on the deformed configuration for a hexagonal mesh composed of 280 cells.

\footnotetext{
${ }^{1}$ https://github.com/wareHHOuse/diskpp
} 


\begin{tabular}{|c|c|c|c|c|c|c|c|c|}
\hline \multirow{2}{*}{$\begin{array}{c}\text { Mesh } \\
\text { size } h\end{array}$} & \multicolumn{2}{|c|}{$k=1$} & \multicolumn{2}{c|}{$k=2$} & \multicolumn{2}{c|}{$k=3$} & \multicolumn{2}{c|}{$k=4$} \\
\cline { 2 - 10 } & $H^{\perp}$-error & order & $H^{1}$-error & order & $H^{1}$-error & order & $H^{1}$-error & order \\
\hline $3.33 \mathrm{e}-1$ & $5.42 \mathrm{e}-3$ & - & $4.41 \mathrm{e}-4$ & - & $5.56 \mathrm{e}-6$ & - & $1.84 \mathrm{e}-7$ & - \\
\hline $1.75 \mathrm{e}-1$ & $1.38 \mathrm{e}-3$ & 2.13 & $5.87 \mathrm{e}-5$ & 3.13 & $3.33 \mathrm{e}-7$ & 4.06 & $5.34 \mathrm{e}-9$ & 5.11 \\
\hline $9.06 \mathrm{e}-2$ & $3.47 \mathrm{e}-4$ & 2.08 & $7.62 \mathrm{e}-6$ & 3.07 & $2.05 \mathrm{e}-8$ & 4.02 & $1.63 \mathrm{e}-10$ & 5.04 \\
\hline $4.60 \mathrm{e}-2$ & $8.70 \mathrm{e}-5$ & 2.05 & $9.72 \mathrm{e}-7$ & 3.04 & $1.28 \mathrm{e}-9$ & 4.00 & $6.66 \mathrm{e}-12$ & 4.61 \\
\hline
\end{tabular}

Table 1: 2D manufactured solution: $H^{1}$-error and convergence order vs. $h$ for $\theta=1$.

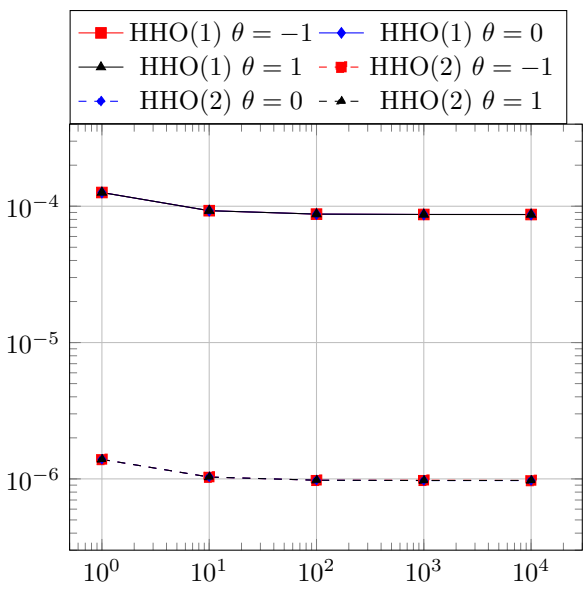

(a) $H^{1}$-error vs. $\lambda\left(\gamma_{n}=\gamma_{t}=2 \mu\right)$

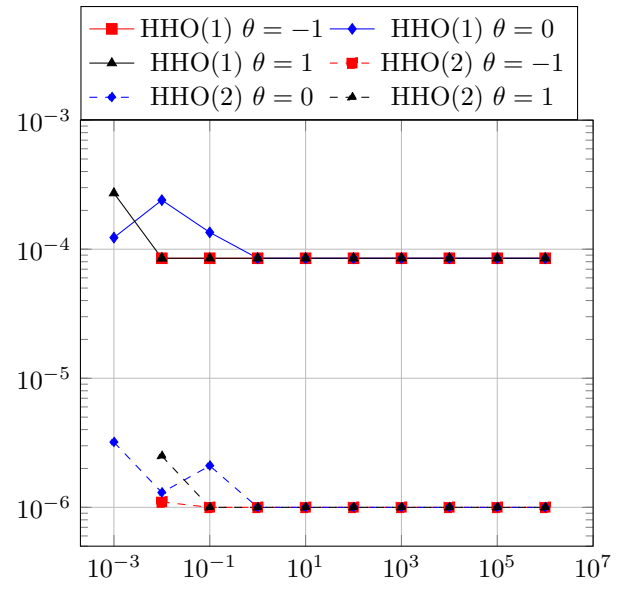

(b) $H^{1}$-error vs. $\frac{\gamma_{n}}{2 \mu}\left(\lambda=1000\right.$ and $\left.\gamma_{t}=2 \mu\right)$

Figure 4: 2D manufactured solution: $H^{1}$-error vs. $\lambda$ and $\frac{\gamma_{n}}{2 \mu}$ for a hexagonal mesh $(h=4.60 \mathrm{e}-2)$.

We first report in Tab. 1 the $H^{1}$-error (that is, the $\mu$-dependent part of the left-hand side in (39)) and convergence rates as a function of the average mesh size $h$ for $k \in\{1, \ldots, 4\}$ on hexagonal mesh sequences and for the symmetric variant with $\theta=1$. For all $k \in\{1, \ldots, 4\}$, the $H^{1}$-error converges with order $(k+1)$ as predicted in Corollary 4.8 (except for $k=4$ and the finest mesh since we are slightly limited here by the numerical precision). The results are similar for the other variants with $\theta \in\{-1,0\}$ (not shown for brevity). These convergence rates are consistent with the predicted rates in Corollary 4.8 (we are not limited by the regularity of the solution in this test case).

In Fig. 4 we report on a fixed hexagonal mesh the $H^{1}$-error as a function of the material parameter $\lambda$ (left panel) and of the penalty parameter $\frac{\gamma_{n}}{2 \mu}$ with $\gamma_{t}=2 \mu$ fixed (right panel) for $k \in\{1,2\}$ and for the three variants with $\theta \in\{-1,0,1\}$. We observe that the $H^{1}$-error is nearly independent of the values of $\lambda$ and $\frac{\gamma_{n}}{2 \mu}$. These results indicate that the HHO-Nitsche method appears to be locking-free in the incompressible limit despite the fact that the theoretical analysis is somewhat more pessimistic. The same comment can be made regarding the necessity to enforce the lower bound in (32).

\section{$5.23 \mathrm{D}$ Hertz contact}

The second benchmark is the well known three-dimensional Hertz contact problem of a half ball in contact with a rigid foundation. The half sphere is centered at the point having coordinates $(0,0,100)$ and has a radius of 100 . The contact boundary is the infinite plane $z:=0$ and a vertical displacement $u_{z}:=-2$ is applied on the top surface. For symmetry reasons, one half of the half ball is discretized. The material parameters are $\mu:=26.9$ and $\lambda:=40.3$. In this benchmark, we modify slightly without difficulty the formulation of Nitsche-HHO method in order to take into account the gap between the contact boundary and the obstacle, since this gap is supposed to be equal to zero in (3) (see, e.g., [12] for the Nitsche-FEM formulation with a nonzero gap). We consider frictionless contact, i.e., $s:=0$, so as to compare our numerical results with a reference solution computed using the mixed quadratic formulation T2-LAC implemented in the industrial software code_aster. For this test case, we do not expect $\mathrm{HHO}(2)$ to deliver a more accurate solution than $\mathrm{HHO}(1)$ since the curved boundary is discretized using tetrahedra with planar faces. We consider only the variant with $\theta=0$ and we set $\gamma_{n}=\gamma_{t}:=2 \mu$. The displacement Euclidean norm on the 
deformed configuration is plotted in Fig. 5a for $\operatorname{HHO}(1)$. In Fig. 5b, we compare for $\operatorname{HHO}(k), k \in\{1,2\}$, and the reference T2-LAC solution, the evolution of the normal component of the Cauchy stress tensor (see (18) for $\mathrm{HHO}$ ) vs. the radial coordinate $r$ at the barycenter of the contact faces. The results for $\mathrm{HHO}(k)$, which are computed on a mesh composed of 3,740 tetrahedra, are in good agreement with the reference solution which is computed on a finer mesh with 102,436 tetrahedra, although some slight differences are visible near $r=15$ where the transition between contact and no-contact occurs.

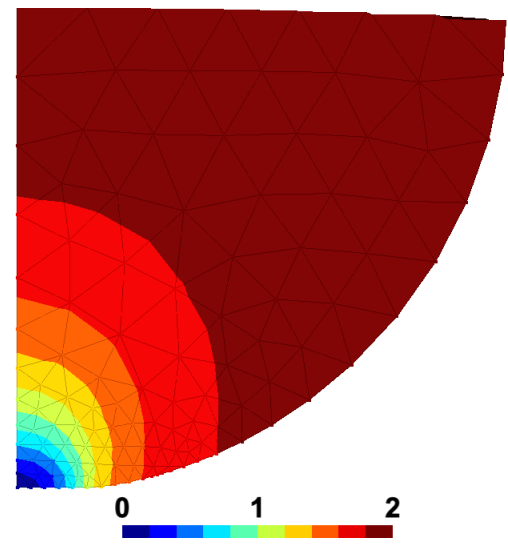

(a) Displacement Euclidean norm on the deformed configuration for $\mathrm{HHO}(1)$

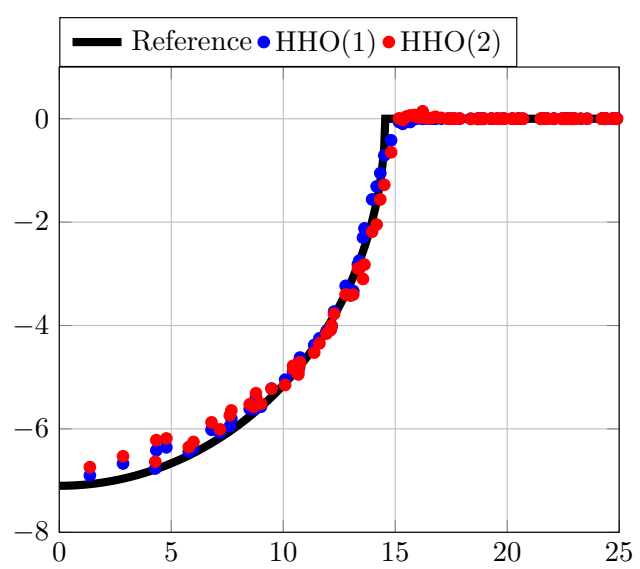

(b) $\sigma_{n, T}^{k}$ vs. radial-coordinate

Figure 5: 3D Hertz contact sphere: displacement Euclidean norm on the deformed configuration and contact pressure vs. radial coordinate.

\subsection{Stick and slip transition (Bostan \& Han test case)}

This third benchmark has been studied previously in [6]. It consists of a rectangular domain $\Omega:=(0,8) \times$ $(0,4)$ which is clamped on the Dirichlet boundary $\Gamma_{\mathrm{D}}:=(0,8) \times\{4\}$ and subjected to a horizontal surface load $g_{\mathrm{N}}:=(400,0)$ on the Neumann boundary $\Gamma_{\mathrm{N}}:=\{0\} \times(0,4)$. The bilateral contact boundary is $\Gamma_{\mathrm{C}}:=(0,8) \times\{0\}$, where a Tresca friction is considered with $s:=150$. Moreover the material parameters are $\mu:=384.6$ and $\lambda:=576.9$. The symmetry parameter is set to $\theta:=1$ and the penalty parameters to $\gamma_{n}=\gamma_{t}:=2 \mu$. The reference solution, referred to as Bostan \& Han, comes from [6, Example 6.2], where a mixed method with adaptive mesh refinement is used.

In Fig. 6 we compare the normalized quantity $\left\|\underline{\sigma}_{t}\right\| / s$ at the barycentrer of the contact faces for $\operatorname{HHO}(k)$, $k \in\{1,2\}$, and the reference solution on two different meshes: a coarse mesh composed of 225 quadrangles and a fine mesh composed of 10,000 quadrangles. We observe that the results for the $\mathrm{HHO}(k)$ methods are close to the reference solution even on the coarse mesh. Moreover, on the fine mesh, both methods accurately capture the transition between slip $\left(\left\|\underline{\sigma}_{t}\right\| / s=1\right)$ and stick $\left(\left\|\underline{\sigma}_{t}\right\| / s<1\right)$ at $x \sim 2.7$. Moreover, the results are slightly more accurate for $\mathrm{HHO}(2)$ than for $\mathrm{HHO}(1)$ on the coarse mesh and quasi-identical on the fine mesh. We note that increasing $k$ does not improve significantly the results as expected, since the regularity of the solution is a limiting factor in this example.

To evaluate the influence of the penalty parameters $\gamma_{n}$ and $\gamma_{t}$, we compare the total number of Newton's iterations needed to solve the nonlinear problem (17) versus the magnitude of the normalized penalty parameter $\gamma_{0}:=\frac{\gamma_{n}}{2 \mu}=\frac{\gamma_{t}}{2 \mu}$. The Newton's iterations are stopped under a relative residual convergence threshold of $10^{-7}$, and convergence failure is reported after 200 iterations. We present the results in Fig. 7 for the coarse mesh composed of 225 quadrangles, the three symmetry variants, and the polynomial degrees $k \in\{1,2\}$. For $\mathrm{HHO}(1)$, we remark that the different symmetry variants need the same total number of Newton's iterations ( 5 here) if $\gamma_{0} \geq 10^{2}$, whereas the skew-symmetric variant $(\theta=-1)$ is the most robust since the number of Newton's iterations is almost independent of $\gamma_{0}$, contrary to the incomplete variant $(\theta=0)$ which suffers some degradation in the convergence for $\gamma_{0} \leq 1$, and to the symmetric variant $(\theta=1)$ which does not converge anymore if $\gamma_{0}<10^{-2}$. For $\operatorname{HHO}(2)$, the skew-symmetric variant $(\theta=-1)$ is again the most robust, as for $\mathrm{HHO}(1)$. However, both variants with $\theta=0$ and $\theta=1$ now exhibit a similar behavior and do not converge anymore if $\gamma_{0}<10^{-3}$ and $\gamma_{0}<10^{-2}$, respectively. Finally, we observe that for 
$\mathrm{HHO}(2)$, the number of iterations increases significantly for $\gamma_{0} \geq 10^{4}$ whatever the value of the symmetry parameter (and do not converge anymore if $\gamma_{0}>10^{5}$ ). This effect is not observed for $\operatorname{HHO}(1)$. To sum up this numerical experiment, an optimal range of values for $\gamma_{0}$ seems to be $10^{-1} \leq \gamma_{0} \leq 10^{3}$.

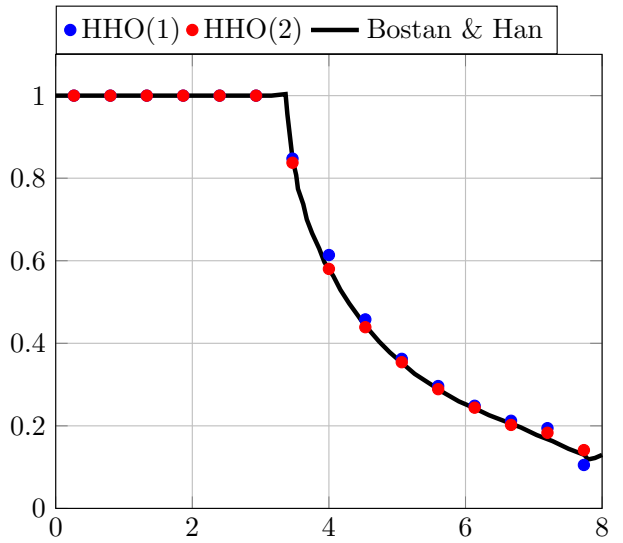

(a) mesh composed of 225 quadrangles

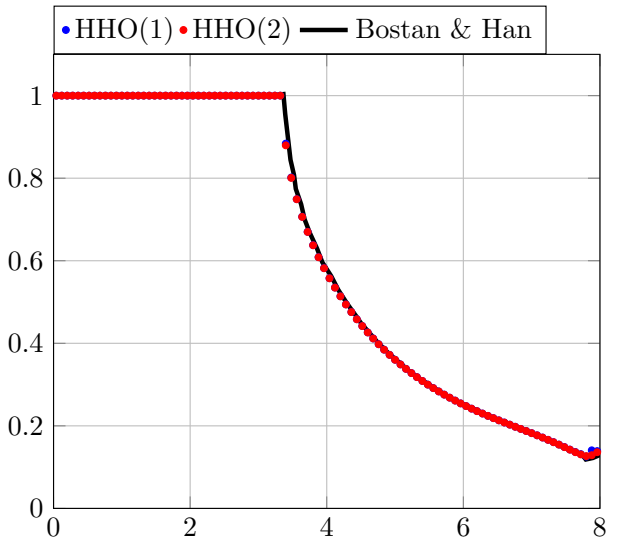

(b) mesh composed of 10,000 quadrangles

Figure 6: Stick and slip transition (Bostan \& Han test case): $\left\|\underline{\sigma}_{t}\right\| / s$ vs. $x$-coordinate at the barycentrer of the contact faces on a coarse and a fine mesh.

\subsection{A prototype for an industrial application}

This prototype simulates the installation of a notched plug in a rigid pipe. The mesh is composed of 21,200 hexahedra and 510 prisms in the reference configuration, see Fig. 10 (for symmetry reasons, only one quarter of the pipe is discretized). The notched plug has a length of $56 \mathrm{~mm}$ and an outer radius of $8 \mathrm{~mm}$. The pipe is supposed to be rigid and has an inner radius of $8.77 \mathrm{~mm}$ (there is an initial gap of $0.77 \mathrm{~mm}$ between the plug and the pipe). The contact zone $\Gamma_{\mathrm{C}}$ with Tresca's friction $(s:=3,000 \mathrm{MPa})$ is between the rigid pipe and the ten notches of the plug. In the actual industrial setting, an indenter imposes a displacement to the upper surface of the plug. To simplify, sufficiently large vertical and horizontal forces are applied to the upper surface of the plug to impose a contact between the pipe and the notches. The material parameters for the plug are $\mu:=80,769 \mathrm{MPa}$ and $\lambda:=121,154 \mathrm{MPa}$ (which correspond to a Young modulus $E:=210,000 \mathrm{MPa}$ and a Poisson ratio $\nu:=0.3)$. The simulation is performed using $\mathrm{HHO}(1)$, the symmetry variant $\theta:=1$, and the penalty parameters $\left.\gamma_{n}=\gamma_{t}:=2 \mu\right)$.

The von Mises stress is plotted in Fig. 9 on the deformed configuration. The maximal value is reached where the force is applied. Moreover, a zoom on the contact zone is plotted in Fig. 9b. We remark that there is contact between the notches and the pipe. Finally, the normal stress $\sigma_{n}$ is visualized in Fig. 10 on the inferior surface of the plug. We remark that all the notches are in contact except the first three (from left to right) and the last one (where $\sigma_{n}=0$ ), and that a transition between contact and non-contact is located at the fourth notch. Moreover, the maximal value of the contact pressure is reached at the extremity of the notches.

\section{Conclusion}

We have devised, analyzed, and evaluated numerically a HHO discretization combined with a Nitsche method to impose weakly contact and Tresca friction conditions in small strain elasticity. We have proved optimal error estimates for this nonlinear problem and have studied the robustness of the estimates in the incompressible limit. The numerical tests indicate that robustness is achieved in all configurations considered herein. This work can be pursued in several directions, such as extending the analysis to Coulomb friction and addressing further extensions (multi-body contact, large transformations, plasticity) for industrial applications. 


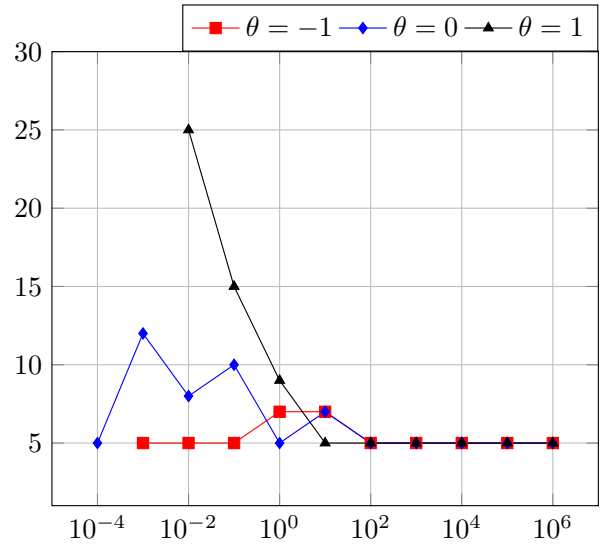

(a) $\operatorname{HHO}(1)$

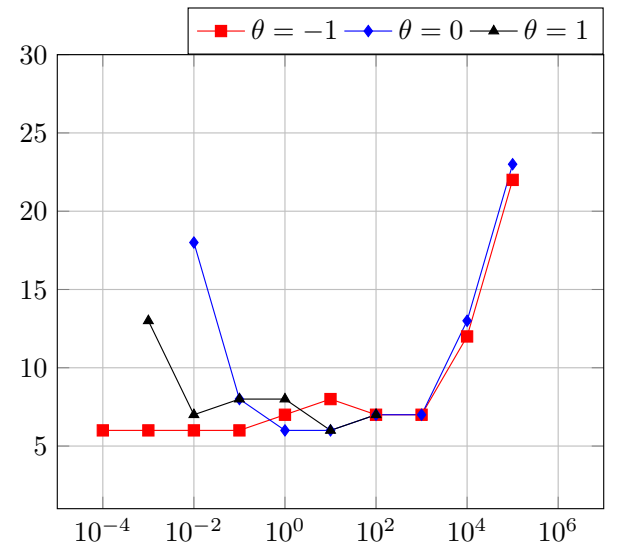

(b) $\mathrm{HHO}(2)$

Figure 7: Stick and slip transition (Bostan \& Han test case): total number of Newton's iterations vs. the normalized penalty parameter $\gamma_{0}$ for a mesh composed of 225 quadrangles (no value is plotted if Newton's method has not converged after 200 iterations).

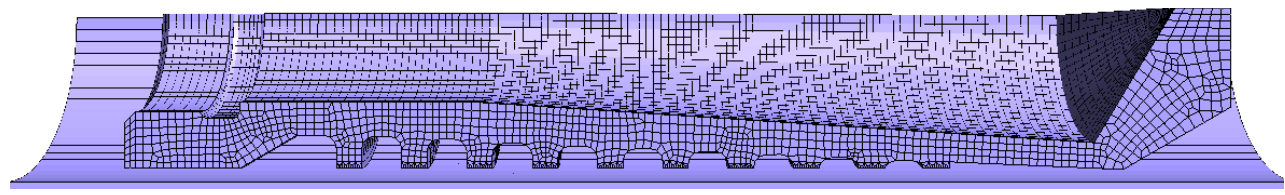

Figure 8: Notch plug : mesh composed of 21,200 hexahedra and 510 prisms in the reference configuration.

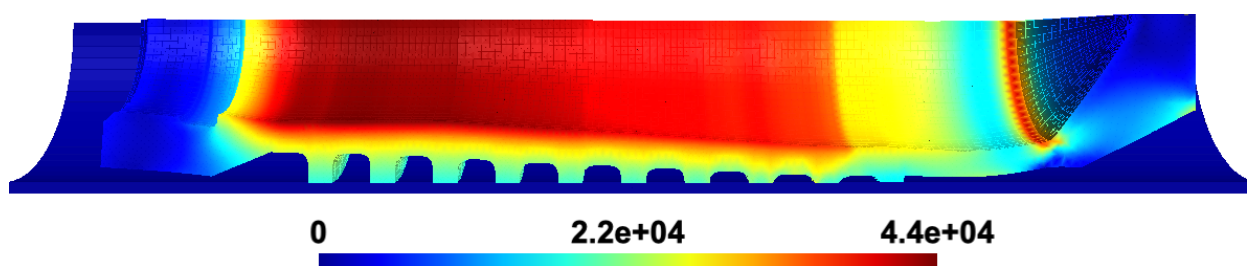

(a) Full structure

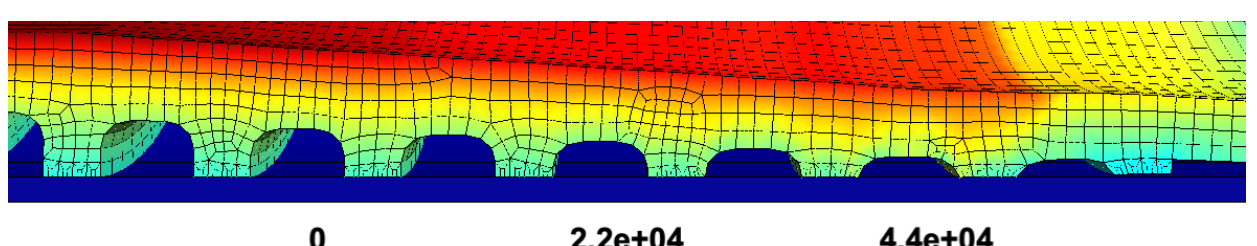

$2.2 e+04$

$4.4 \mathrm{e}+04$

(b) Zoom on the contact zone

Figure 9: Notch plug: von Mises stress on the deformed configuration (in MPa). 


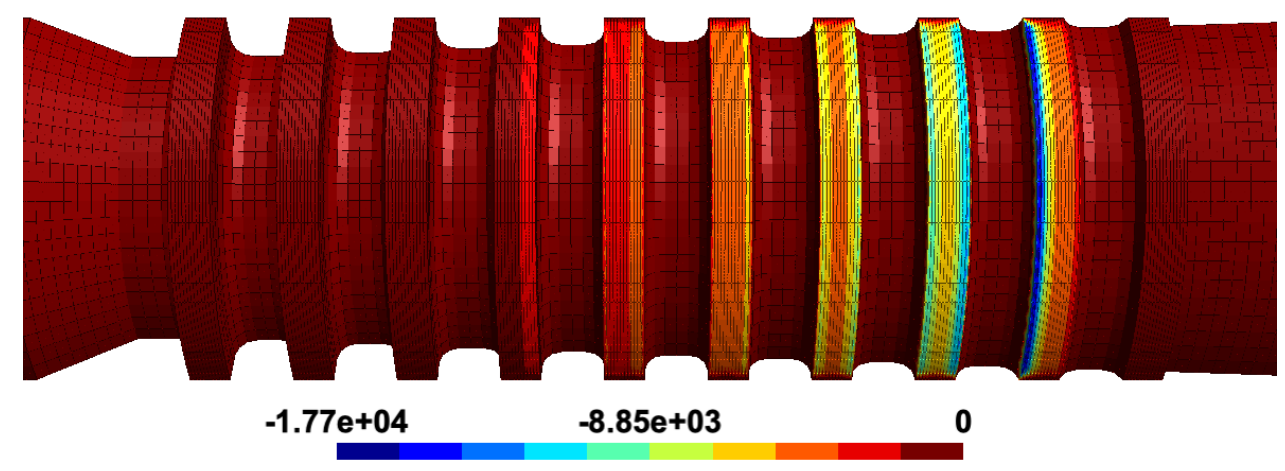

Figure 10: Notch plug: normal stress $\sigma_{n}$ on the contact zone (in MPa).

\section{References}

[1] M. Abbas, G. Drouet, And P. Hild, The local average contact (LAC) method, Comput. Methods Appl. Mech. Engrg., 339 (2018), pp. 488-513.

[2] M. AbBas, A. ERn, And N. Pignet, Hybrid High-Order methods for finite deformations of hyperelastic materials, Comput. Mech., 62 (2018), pp. 909-928.

[3] M. AbBas, A. ERn, And N. Pignet, A Hybrid High-Order method for finite elastoplastic deformations within a logarithmic strain framework, Int. J. Numer. Methods Eng., 120 (2019), pp. 303-327.

[4] M. AbBas, A. ERn, And N. Pignet, A Hybrid High-Order method for incremental associative plasticity with small deformations, Comput. Methods Appl. Mech. Engrg., 346 (2019), pp. 891-912.

[5] B. Ayuso de Dios, K. Lipnikov, and G. Manzini, The nonconforming virtual element method, ESAIM: Math. Model. Numer. Anal. (M2AN), 50 (2016), pp. 879-904.

[6] V. Bostan And W. Han, A posteriori error analysis for finite element solutions of a frictional contact problem, Comput. Methods Appl. Mech. Engrg., 195 (2006), pp. 1252-1274.

[7] M. Botti, D. A. Di Pietro, and P. Sochala, A Hybrid High-Order method for nonlinear elasticity, SIAM J. Numer. Anal., 55 (2017), pp. 2687-2717.

[8] H. Brezis, Équations et inéquations non linéaires dans les espaces vectoriels en dualité, Ann. Inst. Fourier (Grenoble), 18 (1968), pp. 115-175.

[9] E. Burman And A. ERn, An unfitted Hybrid High-Order method for elliptic interface problems, SIAM J. Numer. Anal., 56 (2018), pp. 1525-1546.

[10] K. L. Cascavita, F. ChOuly, And A. ERn, Hybrid High-Order discretizations combined with Nitsche's method for Dirichlet and Signorini boundary conditions, IMA J. Numer. Anal., (2020), https://doi.org/10.1093/imanum/drz038.

[11] F. Chouly, An adaptation of Nitsche's method to the Tresca friction problem, J. Math. Anal. Appl., 411 (2014), pp. 329-339.

[12] F. Chouly, M. Fabre, P. Hild, J. Pousin, And Y. Renard, Residual-based a posteriori error estimation for contact problems approximated by Nitsche's method, IMA J. Numer. Anal., 38 (2018), pp. 921-954.

[13] F. Chouly And P. Hild, A Nitsche-based method for unilateral contact problems: numerical analysis, SIAM J. Numer. Anal., 51 (2013), pp. 1295-1307.

[14] F. Chouly And P. Hild, On convergence of the penalty method for unilateral contact problems, Appl. Numer. Math., 65 (2013), pp. 27-40. 
[15] F. Chouly, P. Hild, And Y. Renard, Symmetric and non-symmetric variants of Nitsche's method for contact problems in elasticity: theory and numerical experiments, Math. Comp., 84 (2015), pp. 10891112.

[16] F. Chouly, R. Mlika, And Y. Renard, An unbiased Nitsche's approximation of the frictional contact between two elastic structures, Numer. Math., 139 (2018), pp. 593-631.

[17] M. Cicuttin, D. A. Di Pietro, And A. Ern, Implementation of Discontinuous Skeletal methods on arbitrary-dimensional, polytopal meshes using generic programming, J. Comput. Appl. Math., 344 (2018), pp. 852-874.

[18] B. Cockburn, D. A. Di Pietro, And A. Ern, Bridging the Hybrid High-Order and Hybridizable discontinuous Galerkin methods, ESAIM Math. Model. Numer. Anal., 50 (2016), pp. 635-650.

[19] B. Cockburn, J. Gopalakrishnan, And R. Lazarov, Unified hybridization of discontinuous Galerkin, mixed, and continuous Galerkin methods for second order elliptic problems, SIAM J. Numer. Anal., 47 (2009), pp. 1319-1365.

[20] A. Curnier And P. Alart, A generalized Newton method for contact problems with friction, J. Méc. Théor. Appl., 7 (1988), pp. 67-82.

[21] D. A. Di Pietro And A. ERn, Mathematical aspects of discontinuous Galerkin methods, vol. 69 of Mathématiques \& Applications, Springer, Heidelberg, 2012.

[22] D. A. Di Pietro And A. ERn, A Hybrid High-Order locking-free method for linear elasticity on general meshes, Comput. Methods Appl. Mech. Engrg., 283 (2015), pp. 1-21.

[23] D. A. Di Pietro, A. ERn, And S. Lemaire, An arbitrary-order and compact-stencil discretization of diffusion on general meshes based on local reconstruction operators, Comput. Methods Appl. Math., 14 (2014), pp. 461-472.

[24] I. Dione, Optimal convergence analysis of the unilateral contact problem with and without Tresca friction conditions by the penalty method, J. Math. Anal. Appl., 472 (2019), pp. 266-284.

[25] G. Drouet And P. Hild, Optimal convergence for discrete variational inequalities modelling Signorini contact in $2 D$ and $3 D$ without additional assumptions on the unknown contact set, SIAM J. Numer. Anal., 53 (2015), pp. 1488-1507.

[26] Electricité De France, Finite element code_aster, structures and thermomechanics analysis for studies and research. Open source on www.code-aster.org, 1989-2019.

[27] A. ERn AND J.-L. Guermond, Finite element quasi-interpolation and best approximation, ESAIM Math. Model. Numer. Anal., 51 (2017), pp. 1367-1385.

[28] Q. Guan, M. GunzBurger, And W. ZhaO, Weak-Galerkin finite element methods for a second-order elliptic variational inequality, Comput. Methods Appl. Mech. Engrg., 337 (2018), pp. 677-688.

[29] P. Hild AND Y. RENARD, An improved a priori error analysis for finite element approximations of Signorini's problem, SIAM J. Numer. Anal., 50 (2012), pp. 2400-2419.

[30] N. KIKUCHI AND J. T. ODEn, Contact problems in elasticity: a study of variational inequalities and finite element methods, vol. 8 of SIAM Studies in Applied Mathematics, Society for Industrial and Applied Mathematics (SIAM), Philadelphia, PA, 1988.

[31] E. Pipping, O. Sander, And R. Kornhuber, Variational formulation of rate- and state-dependent friction problems, ZAMM Z. Angew. Math. Mech., 95 (2015), pp. 377-395.

[32] F. Wang, W. Han, And X. Cheng, Discontinuous Galerkin methods for solving the Signorini problem, IMA J. Numer. Anal., 31 (2011), pp. 1754-1772.

[33] F. Wang And H. Wei, Virtual element method for simplified friction problem, Appl. Math. Letters, 85 (2018), pp. 125-131. 
[34] B. Wohlmuth, Variationally consistent discretization schemes and numerical algorithms for contact problems, Acta Numer., 20 (2011), pp. 569-734.

[35] P. Wriggers, W. T. Rust, And B. D. Reddy, A virtual element method for contact, Comput. Mech., 58 (2016), pp. 1039-1050.

[36] M. Zhao, H. Wu, And C. Xiong, Error analysis of HDG approximations for elliptic variational inequality: obstacle problem, Numer. Algorithms, 81 (2019), pp. 445-463. 